\title{
Generation of High-Amylose Rice through CRISPR/Cas9-Mediated Targeted Mutagenesis of Starch Branching Enzymes
}

\begin{abstract}
Yongwei Sun ${ }^{1 \dagger}$, Guiai Jiao't, Zupei Liu' ${ }^{1 \dagger}$, Xin Zhang', Jingying Li', Xiuping Guo', Wenming $D u^{1}$, Jinlu Du ${ }^{1}$, Frédéric Francis ${ }^{3}$, Yunde Zhao ${ }^{4 *}$ and Lanqin Xia ${ }^{1 *}$

1 Institute of Crop Sciences (ICS), Chinese Academy of Agricultural Sciences (CAAS), Beijing, China, ${ }^{2}$ China National Rice Research Institute, Hangzhou, China, ${ }^{3}$ Functional and Evolutionary Entomology, Gembloux Agro-bio Tech, University of Liege, Gembloux, Belgium, ${ }^{4}$ Section of Cell and Developmental Biology, University of California, San Diego, La Jolla, CA, USA
\end{abstract}

\section{OPEN ACCESS}

Edited by:

Agnieszka Ludwików,

Adam Mickiewicz University

in Poznań, Poland

Reviewed by:

Ibrokhim Abdurakhmonov,

The Center of Genomics

and Bioinformatics, Uzbekistan

Yuriko Osakabe,

University of Tokushima, Japan

${ }^{*}$ Correspondence:

Lanqin Xia

xialanqin@caas.cn

Yunde Zhao

yundezhao@ucsd.edu

${ }^{\dagger}$ These authors have contributed equally to this work.

Specialty section:

This article was submitted to

Plant Biotechnology,

a section of the journal

Frontiers in Plant Science

Received: 19 December 2016 Accepted: 20 February 2017

Published: 07 March 2017

Citation:

Sun YW, Jiao GA, Liu ZP, Zhang $X$

Li JY, Guo XP, Du WM, Du JL,

Francis $F$, Zhao YD and Xia LQ

(2017) Generation of High-Amylose

Rice through CRISPR/Cas9-Mediated

Targeted Mutagenesis of Starch

Branching Enzymes.

Front. Plant Sci. 8:298.

doi: 10.3389/fpls.2017.00298
Cereals high in amylose content (AC) and resistant starch (RS) offer potential health benefits. Previous studies using chemical mutagenesis or RNA interference have demonstrated that starch branching enzyme (SBE) plays a major role in determining the fine structure and physical properties of starch. However, it remains a challenge to control starch branching in commercial lines. Here, we use CRISPR/Cas9 technology to generate targeted mutagenesis in SBEI and SBEllb in rice. The frequencies of obtained homozygous or bi-allelic mutant lines with indels in SBEI and SBEllb in $T_{0}$ generation were from 26.7 to $40 \%$. Mutations in the homozygous $T_{0}$ lines stably transmitted to the $T_{1}$ generation and those in the bi-allelic lines segregated in a Mendelian fashion. Transgene-free plants carrying only the frame-shifted mutagenesis were recovered in $\mathrm{T}_{1}$ generation following segregation. Whereas no obvious differences were observed between the sbel mutants and wild type, sbell mutants showed higher proportion of long chains presented in debranched amylopectin, significantly increased AC and RS content to as higher as 25.0 and $9.8 \%$, respectively, and thus altered fine structure and nutritional properties of starch. Taken together, our results demonstrated for the first time the feasibility to create high-amylose rice through CRISPR/Cas9-mediated editing of SBEllb.

Keywords: amylose content (AC), CRISPR/Cas9, genome editing, resistant starch (RS), rice, starch, starch branching enzyme (SBE)

\section{INTRODUCTION}

Diet-related non-infectious chronic diseases, such as coronary heart disease, diabetes, and certain colon and rectum cancers, are major causes of morbidity and mortality in both developed and developing countries (Regina et al., 2006; Chen et al., 2012). Around 600 million people are estimated to live with diabetes in 2035 (Unwin et al., 2013). Resistant starch (RS) is a kind of starch or starch products that are not digestible and absorbed in the stomach or small intestine and passed on directly to the large intestine (Asp, 1992). A cereal grain higher in amylose content (AC) is always a good source of RS (Jiang et al., 2010). Cereals high in RS are benefit to improve human health and to reduce the risk of those serious non-infectious diseases (Regina et al., 2006). 
Consumption of RS could lead to a decreased glycemic index (GI), which represents the rise of blood sugar level following carbohydrate ingestion, and could also prevent the development of non-reversible insulin resistance in healthy individuals and diabetics, and thus decrease the incidence and mitigate the severity of type II diabetes (Giacco et al., 1998; Hoebler et al., 1999; Vonk et al., 2000). RS also produces short-chain fatty acids (SCFAs) which help to keep colon tissue healthier. The acetic, propionic, and butyric acids which composed the SCFA are beneficial to lower the intestinal $\mathrm{pH}$, inhibit the growth of harmful bacteria, and encourage beneficial bacteria to grow in the bowel (Regmi et al., 2011). Although a high AC diet is highly desirable and healthier food for some consumers, cereal crops higher in AC are not widely available (Zhu et al., 2012). Thus, there is an increasing need to develop cereal crops high in RS to meet rapidly growing challenges in nutrition for public health at population level (Regina et al., 2006; Chen et al., 2012; Li and Gilbert, 2016).

Rice is one of the major staple food crops consumed by nearly half of the world population. Each year, about 4.5 million hectares of rice are grown worldwide. In Asia, rice and its processed products provide $60-70 \%$ daily calories for over 2 billion people (Food and Agriculture Organization [FAO], 2004). And about $90 \%$ of a milled rice kernel is starch. Starch consists of glucose polymers, amylose and amylopectin, which are polymerized through $\alpha-1,4$ and $\alpha-1,6$ linkages. Amylose has infrequent $\alpha-1,6$ linkages and a lower degree of polymerization (DP), whereas amylopectin is a large highly branched polysaccharide with higher DP. In cooked foods, amylose molecules re-associate rapidly to form a precipitate or gel upon cooling, forming complexes which resist digestion, whereas the re-associate rate of amylopectin molecules are slowly and thus the formed complexes are more readily digestible. Therefore, high-amylose products always have higher RS content (Regina et al., 2006). Amylose and amylopectin are synthesized via two different pathways. The synthesis of amylose requires an active granulebound starch synthase (GBSS), whereas amylopectin is a product of a complex pathway which involves different isoforms of starch synthase (SS), starch branching enzymes (SBEs), and starch-debranching enzymes (SDBEs) (Ball and Morell, 2003). SBEs catalyze the cleavage of and transfer of $\alpha-1,4$-linked glucan chain and subsequent conjugate the part of that chain to the 6-hydroxyl groups along another chain, thus produce branches in amylopectin (Syahariza et al., 2013).

Analyses of the ACs of a set of germplasm collected by the International Rice Research Institute showed that AC in wild and cultivated rice ranges from 0 to $30 \%$ depending on the rice variety (Butardo et al., 2008). In rice endosperm, there exist at least three isoforms of SBEs, SBEI, SBEIIa, and SBEIIb. SBEIIa and SBEIIb share $\sim 80 \%$ sequence identity (Mizuno et al., 1992; Nakamura et al., 1992). SBEIIa is prodominantly expressed in leaves, while $S B E I I b$ is mainly expressed in the rice grains (Yamanouchi and Nakamura, 1992; Ohdan et al., 2005; Yamakawa et al., 2007). SBEIIb is a major SBEII isoform in the rice endosperm (Yamanouchi and Nakamura, 1992). To further increase the AC, one can direct the starch synthesis toward amylose production through either overexpressing a suitable GBSS (Waxy) allele
(Hanashiro et al., 1996; Itoh et al., 2003), or suppressing the expression of enzymes involved in amylopectin biosynthesis (Morell and Myers, 2005; Regina et al., 2006; Rahman et al., 2007). High amylose maize, wheat, and barley were achieved through down-regulation of SBEII (Boyer and Preiss, 1978; Satoh et al., 2003; Regina et al., 2006, 2010). In rice, high amylose sbeIIb mutants were generated by means of chemical treatment or radiation (Yano et al., 1985; Kim et al., 2005; Shu et al., 2006), or through hairpin RNA (hp-RNA) mediated RNA interference (RNAi) (Ossowski et al., 2008; Warthmann et al., 2008; Wei et al., 2010; Butardo et al., 2011). However, chemical or physical radiation induced mutations might be accompanied by undesired and uncharacterized mutations in the whole genome (Yano et al., 1985; Asaoka et al., 1986). Furthermore, RNAi-mediated interference of gene expression is often incomplete and the transgene expression varies in different lines. In addition, the transgenic lines are regarded as genetically modified and are subject to costly and time-consuming regulatory processes (Shan et al., 2015).

Genome editing technologies enable precise modifications of DNA sequences in vivo and offer a great promise for harnessing plant genes in crop improvement (Shan et al., 2013; Puchta and Fauser, 2014; Voytas and Gao, 2014; Li et al., 2015; Ma et al., 2015; Svitashev et al., 2015; Endo et al., 2016; Gao et al., 2016; Sun et al., 2016b). CRISPR/Cas9 (Clustered Regularly Interspaced Short Palindromic Repeats/CRISPR-associated Cas9 endonuclease) has revolutionized genome editing because of its specificity, simplicity, and versatility (Cong et al., 2013; Feng et al., 2014; Gao and Zhao, 2014; Zhou et al., 2014; Ma et al., 2015; Wang et al., 2015; Xie et al., 2015; Li J. et al., 2016; Paul and Qi, 2016; Sun et al., 2016a). The Cas9 protein functions as a nuclease and is directed to a target site by an engineered sequence-specific single guide RNA (gRNA). This site-specific targeting is determined by the first 20 nucleotides (target seed sequence) of the gRNA for the DNA recognition (Jinek et al., 2012). CRISPR/Cas9 induces site-specific double-strand breaks (DSBs), which can be then repaired by non-homologous end-joining (NHEJ) or homologous recombination (HR). NHEJ is error-prone and often introduces small insertions or deletions (indels) at cleavage sites, frequently generating frame-shifted knockout mutations (Jinek et al., 2012).

CRISPR/Cas9-mediated gene editing technology has the potential to greatly facilitate plant breeding. However, so far only a very few examples of improvement of agronomic important traits and creation of novel germplasm in crop plants have been reported (Ma et al., 2015; Svitashev et al., 2015; Li M. et al., 2016; Shi et al., 2016; Sun et al., 2016a,b). For example, through CRISPR/Cas9 mediated gene editing, substitution of P165 with Serine in the ALS2 gene using either double-stranded DNA vectors or single-stranded oligonucleotides as repair templates yielded chlorsulfuron resistant maize plants (Svitashev et al., 2015). The replacement of a $5^{\prime}$ untranslated region of the ARGOS 8 gene by a native GOS2 promoter resulted in increased grain yield by five bushels per acre under stress conditions in maize (Shi et al., 2016). In rice, targeted mutagenesis of rice OsWaxy (GBSS) gene by CRISPR/Cas9 led to the AC decreased from 14.6 to $2.6 \%$, a phenotype similar to a natural 
glutinous rice variety (Ma et al., 2015). Mutants of Gn1a, DEP1, GS3, and IPA1 genes, which function as regulators of yield parameters such as grain number, panicle architecture, grain size, and plant architecture, were generated through CRISPR/Cas9mediated targeted mutagenesis, respectively (Li M. et al., 2016). In our previous study, multiple herbicide resistant rice plants were successfully achieved by CRISPR/Cas9-mediated HR for simultaneous in planta substitutions of two amino acid residues in acetolactate synthase (Sun et al., 2016b).

Here, we report the feasibility to create transgene-free high amylose rice plants through CRISPR/Cas9-mediated target gene editing of SBEIIb and SBEI. We defined the roles of SBEI and $S B E I I b$ in determining the AC, fine structure of amylopectin, and physiochemical properties of starch. This work enables the improvement of nutritional properties of starch in rice grain, thus potentially providing health benefits to many people.

\section{MATERIALS AND METHODS}

\section{Construction of the CRISPR/Cas9-Related Vectors}

The vectors used in this study were based on the vector pCXUNCas9 in which the codon-optimized Cas9 was driven by the ubiquitin gene promoter of maize (Zea mays L.). The backbone of pCXUN-Cas9 contains a hygromycin resistant gene (hptII) for callus selection. The PmeI in pCXUN-Cas9 were used for introducing the gRNAs expression cassettes.

The gRNA expression cassettes were synthesized by overlapping PCR. Target-specific sequence of gRNA1 (target $S B E I$ ) or gRNA2 (target SBEIIb) were put at the $5^{\prime}$-end of primers SBEI-F1/SBEI-R1 and SBEIIb-F1/SBEIIb-R1, respectively. Because OsU3 promoter sequences were used in this experiment, we also placed an $\mathrm{A}$ at the first nucleotide of the target sequence. Two PCR reactions were performed by using the plasmid pOsU3-gRNA as a template. The first PCR was performed using primer set hrpme-u3F/SBEI-R1, and the second one used primer set SBEIIb-F1/hrpme-u3R (Supplementary Table S2). Products of PCR 1 and 2 were used as templates for the third PCR reaction with the primer set hrpme-u3F/hrpme-u3R to generate the full length gRNA1 fragment (Supplementary Table S2). The same procedure was used to obtain the gRNA2 fragment by using the primer sets SBEIIb-F1, hrpme-u3R, and hrpme-u3F and SBEIIb-R1 (Supplementary Table S2). At the $5^{\prime}$-end of the primer pairs of hrpme-u3F/ hrpme-u3R, the sequences are homologous to the sequences outsides of Pme I sites in pCXUN-Cas9, respectively. Then, gRNA1 and gRNA2 fragments were cloned to the linearized pCXUN-Cas9 with Pme I, by using pEASY-Uni Seamless Cloning and Assembly Kit (TransGen Biotech, Beijing, China).

\section{Agrobacterium-Mediated Rice Transformation}

The vectors described above were transferred into Agrobacterium tumefaciens strain EHA105 by electroporation. Transformation of rice calli was performed as described by Hiei et al. (1994). The calli derived from japonica cv. Kitaake were selected on media containing $50 \mathrm{mg} / \mathrm{L}$ hygromycin for 4 weeks. Then the vigorously grown calli were transferred to regeneration media to generate green plants.

\section{Molecular Characterization of the Mutant Plants}

Rice genomic DNA was extracted by using a DNA Quick Plant System (TransGen Biotech, Beijing, China). $50 \mathrm{ng}$ of genomic DNA were used as a template to perform PCR amplification using Taq polymerase (Tiangen, Beijing, China). The primer sets RC11-F/RC11-R and RC33-F/RC33-R were designed to flank the designated target sites. The PCR products amplified by the primer sets RC11-F/RC11-R and RC33-F/RC33-R were digested with Cac8 I and Dde I, respectively. Then the undigested band was recovered and directly sequenced to screen for the plants with mutations in SBEI or SBEIIb. The sequence chromatograms were analyzed by a web-based tool ${ }^{1}$ to check the genotype and zygosity of the tested plants. PCR products were also cloned into the TA cloning vector P-easy (TransGen Biotech, Beijing, China), and 10 positive colonies were sequenced for each sample. The PCR primer sets used for the PCR/RE assay and detection of the presence of Cas 9 and gRNA were as listed in Supplementary Table S2. $\mathrm{T}_{1}$ homozygous sbeI and sbeIIb mutant lines derived from either $\mathrm{T}_{0}$ homozygotes or bi-allelic lines were subjected to further analyses.

\section{Measurement of Grain Composition and Molecular Structure of Starch}

The harvested mature panicles were dried at $37^{\circ} \mathrm{C}$ for at least 3 days. Thousand-grain weights ( $g$ ) of selected lines were weighed in triplicate. The SeedCount (SeedCount, Australasia, Pty, Ltd) was used to determine grain appearance and dimensions. The opacity of seeds was investigated using the chalkiness index following the standard of Australian rice industry. Leitz M8 stereomicroscope was used to take photomicrographs of whole brown grain samples.

The granular morphology of starch was investigated by scanning electron microscopy (SEM). Grain samples were transversely cut and mounted on circular aluminum stubs with double-sided sticky tape. Samples were examined and photographed by FEI Quanta 450 (FEI Company, Hillsboro, OR, USA).

The total starch, AC and $\mathrm{RS}$ in the brown rice flour were measured with the starch assay kits Megazyme K-STAR, K-AMYL and K-RSTAR (Megazyme, Wicklow, Ireland) ${ }^{2}$.

To analyze the chain length distributions (CLDs) of amylopectin, $25 \mathrm{mg}$ of rice powder was digested with isoamylase (Megazyme, Wicklow, Ireland ${ }^{2}$ ) at $37^{\circ} \mathrm{C}$ for $24 \mathrm{~h}$ for release of the N-linked oligosaccharides. After release, the oligosaccharides were labeled with a fluorophore called 8-aminopyrene-1, 3, 6, 6trisulfonate. For measurement of the stoichiometry, each labeling reaction is composed of one APTS molecule per molecule of

\footnotetext{
${ }^{1}$ http://dsdecode.scgene.com/

${ }^{2}$ http://www.megazyme.com
} 
oligosaccharide. Added maltose quantitative control/mobility marker and then analyzed by capillary electrophoresis (PA800 plus pharmaceutical analysis system, Carbohydrate labeling and analysis, Beckman Coulter, America) ${ }^{3}$.

\section{Measurement of Pasting and Gelatinization Properties}

Three grams of brown rice flour $(0.5 \mathrm{~mm}$ or less, $14 \%$ moisture basis) was put in $25 \mathrm{ml}$ of distilled water and mixed. The sample was measured by Rapid Visco Analyzer (RVA Techmaster, Newport Scientific, Narrabeen, NSW, Australia), according to the America Association of Cereal Chemists (AACC) Approved Method 61-02.01 (AACC, 1995). The gelatinization of rice flour in urea solutions was determined as described by Nishi et al. (2001).

The sample which finished RVA analysis was cool down to room temperature, then stored at $4^{\circ} \mathrm{C}$ and relative humidity $75 \%$ for $24 \mathrm{~h}$ for retrogradation. The retrograded cooked rice flour was performed texture profile analysis (TPA) analysis. The hardness, gumminess and chewiness were measured using a texture analyzer with $25 \mathrm{~N}$ load cell with a two-cycle compression (TMS-Pro, Food technology corporation, American). The deformation level was $70 \%$ of original sample height and the partly broken rice was compressed again.

For the gelatinization temperature and enthalpy analysis, $5 \mathrm{mg}$ of brown rice flour was mixed with $10 \mu \mathrm{L}$ of distilled water and placed inside an aluminum sample cup, and sealed, and then the samples were analyzed by a differential scanning calorimeter (DSC1 STARe system, METTLER TOLEDO, Switzerland).

\section{Measurement of Reducing Sugar and Pentosan}

The in vitro digestibility of rice flour was determined as described by Englyst et al. (1992) with minor modifications. The rice flour $50 \mathrm{mg}$ weighed in screw-capped tubes, $4 \mathrm{ml}$ of sodium acetate buffer $(0.5 \mathrm{M}, \mathrm{pH}=5.2)$ and $1 \mathrm{ml}(3 \mathrm{U} / \mathrm{ml})$ pancreatic $\alpha$-amylase (Megazyme, Wicklow, Ireland) were added to each tube. The sample was incubated in a shaking water bath $(200 \mathrm{rpm})$ at $37^{\circ} \mathrm{C}$. Aliquots $(0.5 \mathrm{ml})$ were taken at intervals and mixed with $0.5 \mathrm{ml}(1.2 \mathrm{M})$ of acetic acid to terminate the reaction. After centrifugation (2000 rpm, $10 \mathrm{~min}$ ), the reducing sugar content released in the supernatant from each sample was measured using 3,5-dinitrosalicylic acid. Total pentosan content was examined as described by Butardo et al. (2011).

\section{Statistical Analysis}

Grain morphology and starch physiochemical properties of different mutant lines were analyzed by using a one-way variance analysis (ANOVA). The differences were examined using a Student's $t$-test. Significance ( $P$-value) was evaluated at the 1 or $5 \%$ level for all comparisons. For each treatment, the standard error of the mean (SEM) was calculated based on at least three biological replicates. For the $\chi^{2}$ test, $P>0.5$ were considered to be very good agreement with expected segregation ratio.

${ }^{3}$ http://www.beckmancoulter.com/

\section{RESULTS}

\section{CRISPR/Cas9-Mediated Targeted Mutagenesis of SBEI and SBEIIb in Rice}

We targeted the first exon of SBEI (GenBank Accession no GQ150904.1) and the third exon of SBEIIb (GenBank Accession no GQ150916.1) (Figure 1A). Both targets contained restriction sites that were used for screening mutations using PCR-based restriction enzyme (PCR/RE) digestion assay (Figure 1A). We placed the gRNA cassette into the binary vector pCXUN (Figure 1B). We transformed the CRISPR/Cas9 vectors into rice embryogenic calli through Agrobacteriummediated transformation, and generated transgenic lines after two rounds of hygromycin selection. In total, we obtained 32 sbeI mutant rice plants from 40 hygromycin-resistant transgenic plants. Our PCR/RE assay and sequence analyses indicated that, of these 40 transgenic plants, 12.5, 27.5, and $40.0 \%$ were heterozygous, bi-allelic, and homozygous sbeI mutant lines, respectively (Figures $\mathbf{1 C}, \mathbf{E}$ and Table 1). For SBEIIb, 21 sbeIIb mutant plants were recovered from 30 independent transgenic plants regenerated, with a frequency of 6.7, 36.6, and $26.7 \%$ for heterozygous, bi-allelic, and homozygous lines, respectively (Figures $\mathbf{1 D}, \mathbf{F}$ and Table 1). Consistent with previous CRISPR/Cas9 studies (Feng et al., 2014), we found that the majorities of the mutations identified in the mutant lines were small deletions or insertions near the target sites (Figures 1E,F and Table 1). However, some mutants harbored large insertions such as the sbeI-c28 and sbeIIb-c3 mutant lines, which had a 64 and 248 bp insertion at the target sites, respectively (Figures 1E,F). Interestingly, the inserted sequences were derived from the vector used in this study in these both cases.

We further investigated if off-target effects occurred in our experiments. Based on the predictions of the CRISPR-P tool ${ }^{4}$, we identified the potential off-target sites for SBEI and SBEIIb genes. We used site-specific genomic PCR and Sanger sequencing to determine whether the predicted off-target sites were also edited. As shown in Supplementary Table S1, no mutations were detected at the putative off-target loci in rice genome in $\mathrm{T}_{0}$ and $\mathrm{T}_{1}$ offspring of these obtained mutant lines.

\section{Inheritance and Stability of the Identified Mutations and Generation of Transgene-Free Mutant Rice Lines}

To investigate whether the mutations generated through CRISPR/Cas9-mediated editing were heritable, we analyzed the segregation patterns at $\mathrm{T}_{1}$ generation. $\mathrm{T}_{0}$ mutant plants were selfpollinated and the resulting $\mathrm{T}_{1}$ plants were used for segregation analysis (Table 2). We randomly selected 15 to $37 \mathrm{~T}_{1}$ progenies derived from each $\mathrm{T}_{0}$ plant for genotyping analysis (Table 2). We found that all of the mutations detected in $\mathrm{T}_{0}$ plants were transmitted to the $\mathrm{T}_{1}$ generation without the occurrence of new mutations (data not shown). All $\mathrm{T}_{1}$ plants from $\mathrm{T}_{0}$ homozygotes were homozygous for the same mutations. Bi-allelic mutations in $\mathrm{T}_{0}$ plants were apparently transmitted to $\mathrm{T}_{1}$ generation in a

${ }^{4}$ http://crispr.dbcls.jp/ 


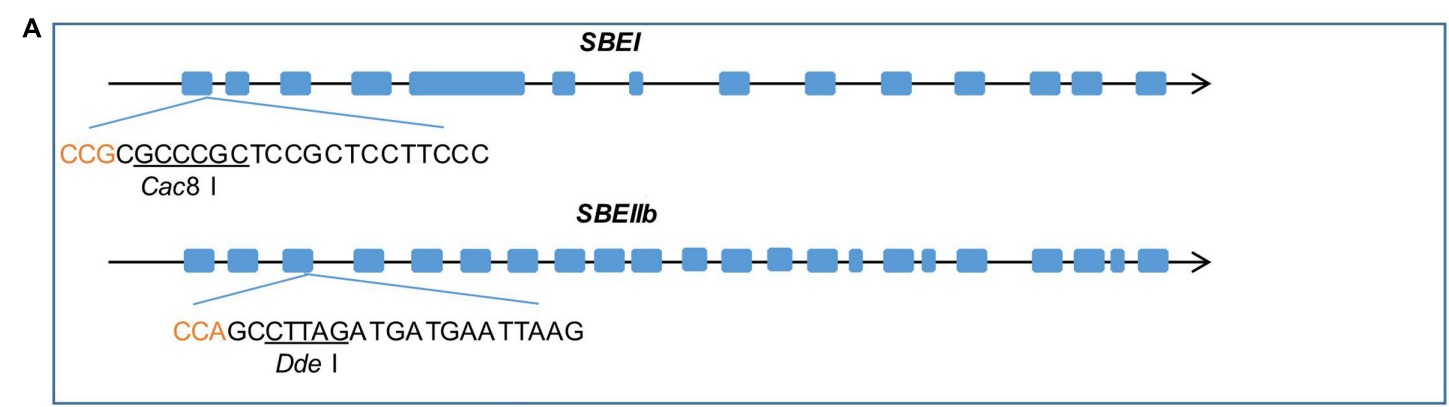

B

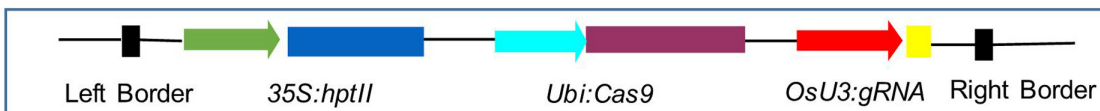

C
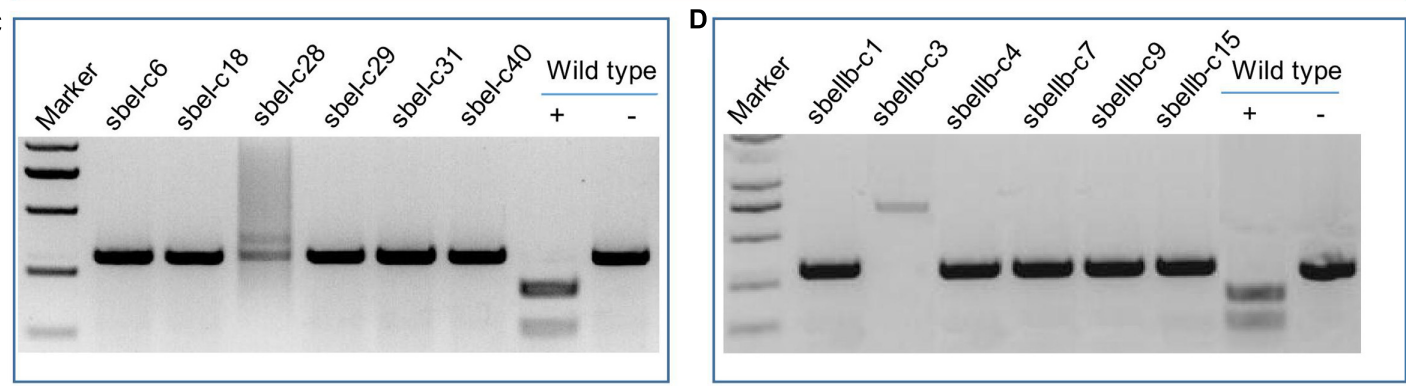

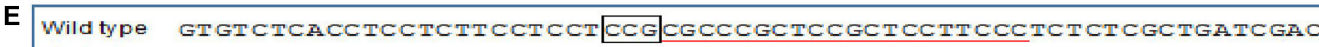

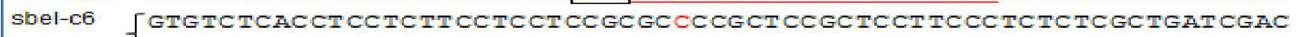
sbel-c18 GTGTCTCACCTCCTCTTCCTCCTCCGCGCTCCGCTCCGCTCCTTCCСTCTCTCGCTGATCGAC sbel-C28 [GTGTCTCACCTCCTCTTCCTCCTCCGCGCTCCGCTCCGCTCCTTCCCTCTCTCGCTGATCGAC -GTGTCTCACCTCCTCTTCCTCCTCCGCGC/CCGCTCCGCTCCTTCCCTCTCTCGCTGATCGAC CCICACCCCGARCIICAAGTCCAACI ICGACCICGCCGAGGACGCCARGCICCAGCTIICCAAG

Sbel-C29 GTGTCTCACCTCCTCTTCTCCTCCGCG-CCGCTCCGCTCCTTCCCTCTCTCGCTGATCGA

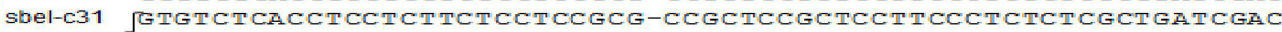
LGTGтCTCACCTCCTCTтCCTCCTCCGCG-------ССтCСтTCCCTCTCTCGCTGATCGAC sbel-C40] [GTGTCTCACCTCCTCTтCстCCтCCGCGCTCCGCTCCGCTCCTтCсCTCTCTCGCTGATCGA

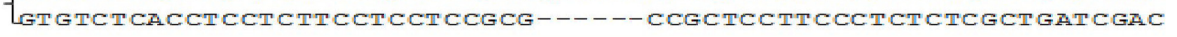

+1
+1
+1
+1
+64

-1
-1
-8
+1
-6

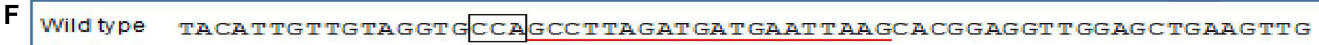
sbellb-c1 TACATTGTTGTAGGTGCCAGCCGTTAGATGATGAATTAAGCACGGAGGTTGGAGCTGAAGTTG +1 sbellb-c3 TACATTGTTGTAGGTGC-----/TTAGATGATGAATTAAGCACGGAGGTTGGAGCTGAAGTTG -5/+248 GAAGTGCT CAT CATTGGAAAACGTTCTTCGGGGCGAAAACTCT CAAGGAT CTTACCGCTGTTGA GATCCAGTTCGATGTAACCCACTCGTGCACCCAACTGATCTTCAGCATCTTTTACTTTCACCAG CGTTTCTGGGT GAGCAAAAACAGGAAGGCAAAATGCCGCAAAAAAGGGAATAAGGGGACACGG

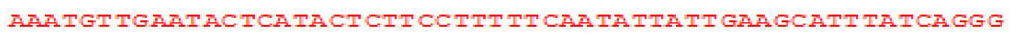

sbellb-C4 TACATTGTTGTAGGTGCCAGCCATTAGATGATGAATTAAGCACGGAGGTTGGAGCTGAAGTTG +1 sbellb-c7 [TACAтTGTTGTAGGTGCCAGCCATTAGATGATGAATtAAGCACGGAGGTTGGAGCTGAAGTTG +1 - $2 A C A T T$ TT GTAGGTCCAGCCGTTAGATGATGAATTAAGCACGGAGGTTGGAGCTGAAGTTG +1 sbellb-c9 TACATTGTTGTAGGTGCCAGCCTTTAGATGATGAATTAAGCACGGAGGTTGGAGCTGAAGTTG +1 Sbellb-c15 [TACATTGTTGTAGGTGCCAGCCGTTAGATGATGAATTAAGCAGGGAGGTTGGAGCTGAAGTTG +1 TACATTGTTGTAGGTGCCAGCCTTTAGATGATGAATTAAGCACGGAGGTTGGAGCTGAAGTTG +1

FIGURE 1 | CRISPR/Cas9 induced mutations in the SBEI and SBEIlb genes. (A) A schematic map of the gRNA target sites on the genomic regions of SBEI and SBEllb. Introns are shown as lines; exons shown as boxes; The PAM motif (CCN) is shown in red; the recognition sites of the enzymes Cac8 I and Dde I are underlined. (B) Schematic presentation of the T-DNA structure in CRISPR/Cas9-mediated genome editing construct. (C,D) Detection of mutations in SBEI and SBEIllb via PCR/RE assay in $T_{0}$ generation. The PCR products of sbel and sbelllb mutant lines are resistant to Cac8 I and Dde I digestion, respectively. As for wild type, '+' means the PCR products of wild type DNA digested by Cac8 I or Dde I; '-' means without digestion. (E,F) Sequencing results of the sbel and sbellb mutant lines, the PAM motifs are boxed, target sequences are underlined, insertions are highlighted in red, dashes indicate deletions. The sbel-c18, sbel-c29, sbellb-c1, sbellb-c3, sbellb-c4, and sbellb-c9 are homozygous lines; the sbel-c6, sbel-c28, sbel-c31, sbel-c40, sbellb-c7, and sbellb-c15 are bi-allelic lines.

Mendelian fashion. For example, sbeI-c31 was a bi-allelic line with a 1 bp deletion (d1) and an 8 bp deletion (d8). Among the $37 \mathrm{~T}_{1}$ plants analyzed, 9 contained $\mathrm{d} 1,17$ were bi-allelic (d1/d8), and 11 were $\mathrm{d} 8$, consistent with the predicted Mendelian segregation ( $\chi^{2}$ test, $P>0.05$ ). We also noticed some rare segregation patterns. For example, mutations in $s b e I I b-c 3$ showed 
TABLE 1 | CRISPR/Cas9-mediated target mutations in SBEI and SBEIlb in rice.

\begin{tabular}{|c|c|c|c|c|c|}
\hline \multirow[t]{2}{*}{ Target gene } & \multirow[t]{2}{*}{ No. of $T_{0}$ plant tested } & \multirow[t]{2}{*}{ Non-edited plants (\%) } & \multicolumn{3}{|c|}{ Zygosity } \\
\hline & & & Heterozygote (\%) & Bi-allelic (\%) & Homozygote (\%) \\
\hline SBEI & 40 & $8(20.0)$ & 5 (12.5) & $11(27.5)$ & $16(40)$ \\
\hline SBEIlb & 30 & $9(30.0)$ & $2(6.7)$ & $11(36.7)$ & $9(26.7)$ \\
\hline
\end{tabular}

TABLE 2 | Transmission and segregation of CRISPR/Cas9-mediated target mutagenesis and transgenes from $T_{0}$ to $T_{1}$ generation.

\begin{tabular}{|c|c|c|c|c|c|c|c|c|}
\hline \multirow[t]{2}{*}{$T_{0}$ line } & \multicolumn{3}{|c|}{$T_{0}$ plants } & \multirow[b]{2}{*}{$\begin{array}{l}\text { Number of } T_{1} \\
\text { plants tested }\end{array}$} & \multicolumn{4}{|c|}{ Segregation of mutations and T-DNA in $T_{1}$ progenies } \\
\hline & Genotype & Zygosity & $\begin{array}{c}\text { Cas } 9 / g R N A / \\
\text { hptll }\end{array}$ & & Targeted mutations & $\begin{array}{c}\text { Expected } \\
\text { segregation ratio }\end{array}$ & $P$-value & Cas9/gRNA/hptll \\
\hline sbel-c6 & $\mathrm{i} 1 \mathrm{a} / \mathrm{i} 1 \mathrm{~b}$ & Bi-allelic & + & 26 & 6 i1a, 12 i1a/i1b, 8 i1b & $1: 2: 1$ & 0.97 & $24+: 2-$ \\
\hline sbel-c18 & i1 & Ho & + & 15 & $15 \mathrm{i} 1$ & N.A & N.A & $15+: 0-$ \\
\hline sbel-c28 & $\mathrm{i} 1 / \mathrm{i} 64$ & Bi-allelic & + & 34 & 17 i1, 11 i1/i64, 6 i64 & $1: 2: 1$ & 0.64 & $26+: 8-$ \\
\hline sbel-c29 & d1 & $\mathrm{Ho}$ & + & 15 & $15 \mathrm{~d} 11$ & N.A & N.A & $15+: 0-$ \\
\hline sbel-c31 & $\mathrm{d} 1 / \mathrm{d} 8$ & Bi-allelic & + & 37 & $9 \mathrm{~d} 1,17 \mathrm{~d} 1 / \mathrm{d} 8,11 \mathrm{~d} 8$ & $1: 2: 1$ & 0.98 & $28+: 9-$ \\
\hline sbel-c40 & $i 1 / d 6$ & Bi-allelic & - & 35 & $10 i 1,17$ i1/d6, 8 d6 & $1: 2: 1$ & 0.99 & $0+: 35-$ \\
\hline sbellb-c1 & i1 & $\mathrm{Ho}$ & + & 22 & $22 \mathrm{i1}$ & N.A & N.A & $17+: 5-$ \\
\hline sbellb-c3 & d5/i248 & $\mathrm{Ho}$ & + & 38 & 38 (d5/i248) & N.A & N.A & $17+: 21-$ \\
\hline sbellb-c4 & i1 & $\mathrm{Ho}$ & + & 17 & $17 \mathrm{i} 1$ & N.A & N.A & $17+: 0-$ \\
\hline sbellb-c7 & i1a/i1b & Bi-allelic & + & 36 & 9 i1a, 20 i1a/i1b, 7 i1b & $1: 2: 1$ & 0.96 & $36+: 0-$ \\
\hline sbellb-c9 & i1 & $\mathrm{Ho}$ & + & 15 & $15 \mathrm{i} 1$ & N.A & N.A & $15+: 0-$ \\
\hline sbellb-c15 & i1a/i1b & Bi-allelic & + & 19 & $5 \mathrm{i} 1 \mathrm{a}, 8 \mathrm{i} 1 \mathrm{a} / \mathrm{i} 1 \mathrm{~b}, 6 \mathrm{i} 1 \mathrm{~b}$ & $1: 2: 1$ & 0.95 & $18+: 1-$ \\
\hline
\end{tabular}

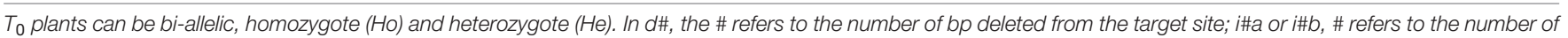

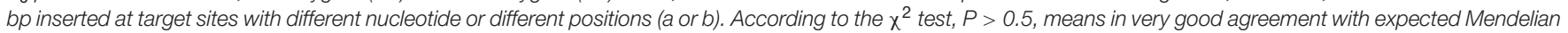

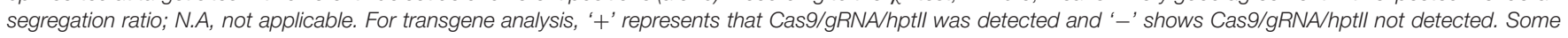

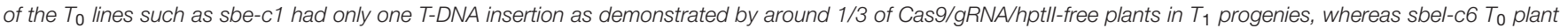

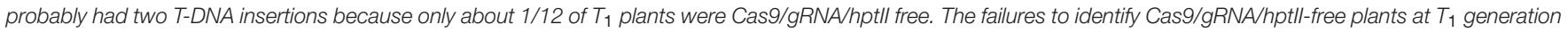
for some lines were probably due to either multiple insertions or tight linkage to the mutations.

a non-Mendelian segregation ratio of $1: 1$, which may be caused by aberrant gamete or seed formation.

To determine whether plasmid DNA sequences, especially Cas9, gRNAs, and hptII sequences, were present in these mutant lines, we performed PCR amplification using the primer sets designed to specifically amplify Cas $9, g R N A s$, and hptII sequences, respectively (Figure $\mathbf{2 A}$ and Supplementary Table S2). Among the $12 \mathrm{~T}_{0}$ mutant lines analyzed, 11 contained gRNAs and Cas 9 sequences. We were able to recover transgene-free mutants at $\mathrm{T}_{1}$ generation. Interestingly, sbeI-c40 mutant line did not contain Cas9, $g R N A$, and $h p t I I$ sequences at both $\mathrm{T}_{0}$ and $\mathrm{T}_{1}$ generations, suggesting that this line escaped from hygromycin selection and the mutations in this line were generated through transient expression of Cas $9 / g R N A$. We also noticed that the indels in sbeIIb-c3 mutant line was homozygous and was caused by an insertion of part of the pCXUN-Cas9 backbone sequences; therefore, these plants are not suitable for future applications. SbeI-c28 was a bi-allelic line with a 1 bp deletion (d1) and a $64 \mathrm{bp}$ insertion (i64) which was derived from pCXUN-Cas9 backbone sequences. However, we were able to recover homozygous plants with only 1 bp of deletion and transgene-free $\mathrm{T}_{1}$ plants (Table 2). Among the rest nine $\mathrm{T}_{0}$ lines tested, Cas $9 / g R N A$ and $h p t I I$ transgene-free plants were successfully recovered from four $\mathrm{T}_{0}$ mutant lines such as two lines for SBEI (sbeI-c6 and sbeI-c31) and another two lines for SBEIIb (sbeIIb-c1 and sbeIIb-c15), in the
$\mathrm{T}_{1}$ generation following segregation (Figures $2 \mathrm{~B}, \mathbf{C}$ and Table 2), indicating that transgene-free mutants could be generated with targeted mutagenesis in SBEI and SBEIIb in rice even in a single generation. More transgene-free plants will be expected in the following generations given that the transgene will segregate following a Mendelian model.

\section{Effects of sbe Mutations on Morphology of Grain and Starch Granule}

Brown grains of $12 \mathrm{~T}_{1}$ homozygous sbeI and sbeIIb mutant lines derived from either $\mathrm{T}_{0}$ homozygotes or bi-allelic lines were used to analyze the grain and starch granule morphology. Brown grains of sbeIIb mutant lines were opaque throughout, while the sbeI mutant lines were chalky. In contrast, grains of wild type appeared uniformly translucent (Figures 3A,B). The weight, width, and thickness of grains from sbeIIb mutant lines were significantly lower than those of wild type, whereas no significant differences were observed between sbeI mutant lines and wild type control (Table 3). It is worth to mention that we did not observe obvious starch granule morphological changes in the grains of $\mathrm{T}_{1}$ homozygous plants of sbeI-c40 with 6 bp deletion (d6), which were progenies from $\mathrm{T}_{0}$ sbeI-c40 bi-allelic line with i1/d6 mutations, indicating that the in-frame deletion of six nucleotides is likely to still produce functional SBEI protein. However, obvious alternations in both the appearance of brown 
A

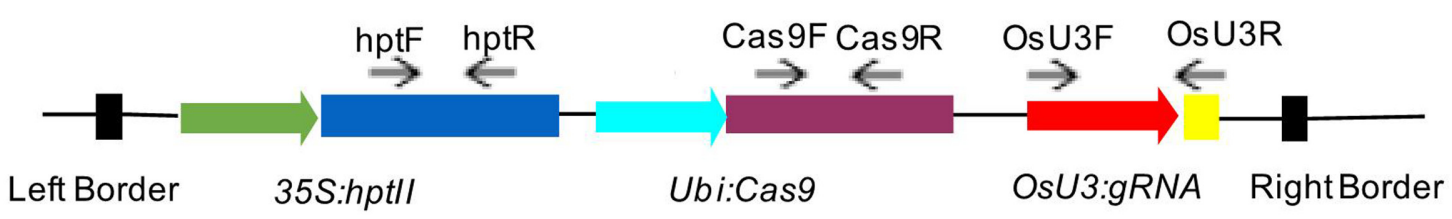

B

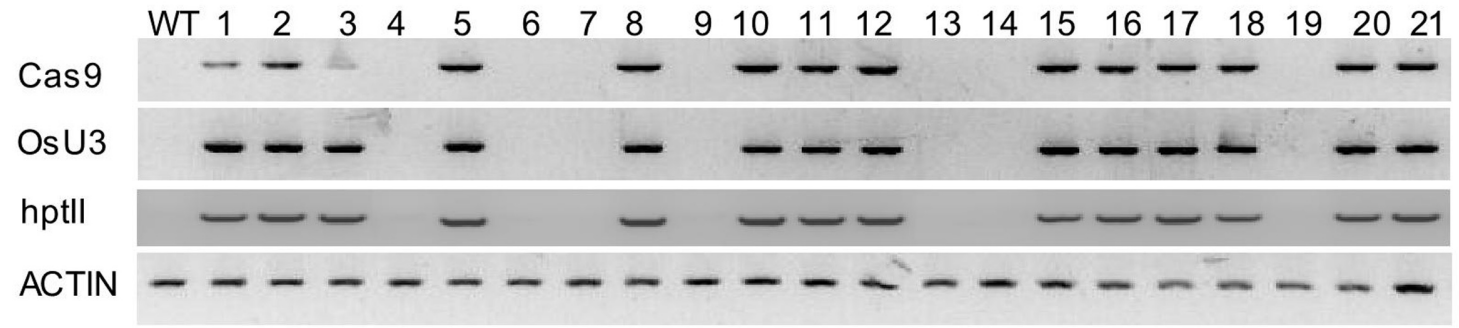

C

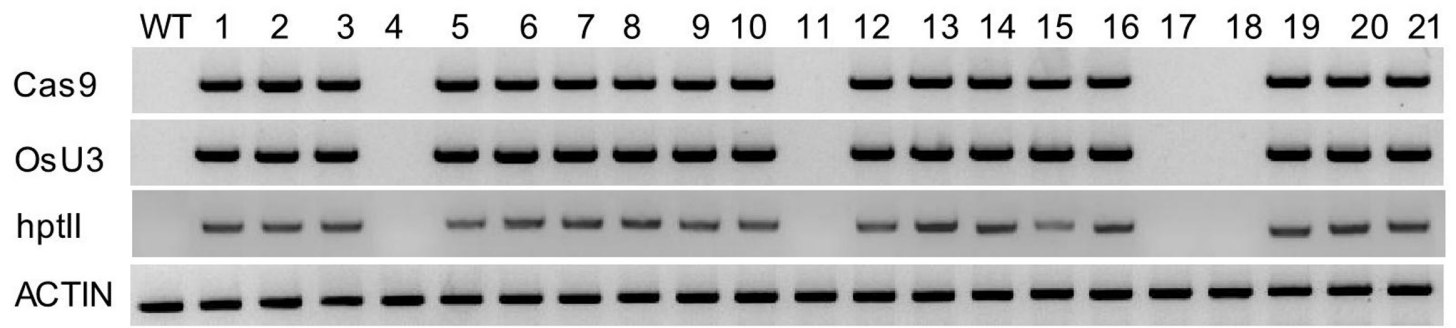

FIGURE 2 | Isolation of transgene-free $\mathbf{T}_{1}$ plants from the sbel-c31 and sbellb-c1 mutant lines. (A) A schematic description of the location/direction of the primer sets used for the analysis for the presence of T-DNA sequences in $T_{1}$ plants of sbel-c31 (B) and sbellb-c1 lines (C) $T_{0}$ lines may contain CRISPR/Cas9 complex in their genome. However, self-pollination of $T_{0}$ lines allows the removal of the T-DNA following segregation in the $T_{1}$ generation plants. DNA fragments of OsU3:gRNA, Cas9 and hptll were not detected in some $T_{1}$ plants of sbel-c31 lines (Number 4, 6, 7, 13, 14, and 19) (B) and $\mathrm{T}_{1}$ plants of sbellb-c1 (Number 4, 11 , 17 and 18) (C), respectively. The control PCR product was amplified from the endogenous ACTIN gene, indicating that the genomic DNAs used have sufficient quality for PCR. WT, wild type DNA control.

grains and morphology of the starch granules in comparison with the wild type were observed in a $\mathrm{T}_{1}$ homozygous line sbeIIb-c3 with $\mathrm{d} 5 /$ i2 248 mutations (Figure $\mathbf{1 F}$ ), probably because the large in-frame indels (deletions or insertions) disrupted the function of target gene.

We also analyzed the morphology of starch granules using SEM. Starch granules from sbeIIb mutant lines were heterogeneous in size and shape, with large spaces in between, and without compound granular organization. The starch granules from sbeI lines were big, rounded, and irregularly arranged. In contrast, the starch granules of wild type were compact, compound, and angular (Figure 3C). Changes in starch granule morphology were more pronounced in sbeIIb mutant lines than that in sbeI lines. The surfaces of both wild type and the sbeI mutant granules were smooth with no or few structural debris (Figure 3C).

\section{Impact of sbe Mutations on Starch Structures}

The total starch contents in the polished grains of both sbeI and sbeIIb mutant lines were comparable to that of the wild type (Figure 4A). However, sbeIIb mutant lines produced grains with much more amylose compared to sbeI mutant lines and wild type (25 vs. $15 \%$ ) (Student's $t$-test, ${ }^{* *} P<0.01$ ) (Figure $4 B$ ). The ratio of amylose/amylopectin was also increased significantly in sbeIIb mutant lines (Student's $t$-test, ${ }^{* *} P<0.01$ ) (Figure 4C). More importantly, all sbeIIb mutant lines displayed altered CLDs of debranched starches. The grains of sbeIIb mutant lines had a significant increase in the ratio of amylopectin longer chains with DP higher than 14 (DP > 14) and a concomitant decrease in the short chains (DP 6-12). For the sbeI mutant lines, we observed a slight increase in the ratio of amylopectin short chains (DP 6-12) followed by a decrease in the longer chains (DP $>14$ ), when compared with the wild type control (Figure 5). The decrease of short chains was pronounced in sbeIIb mutant lines (>3\% for DP 9), whereas the increase reached up to $1 \%$ for DP 18 (Figure 5B).

\section{Physicochemical and Nutritional Properties of Starch}

The changes of starch granule morphology and fine structure of amylopectin resulted in the alternations of physicochemical and nutritional properties of starch. In comparison with the wild type control, the polished grains of sbeI and sbeIIb mutant 
A
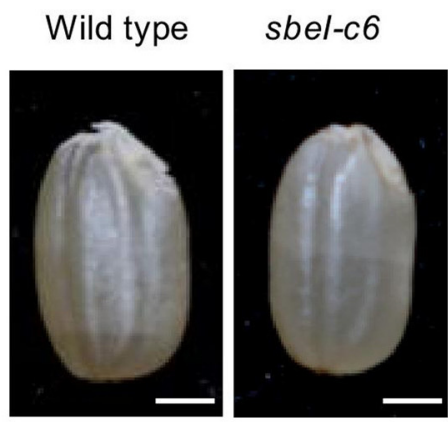

C

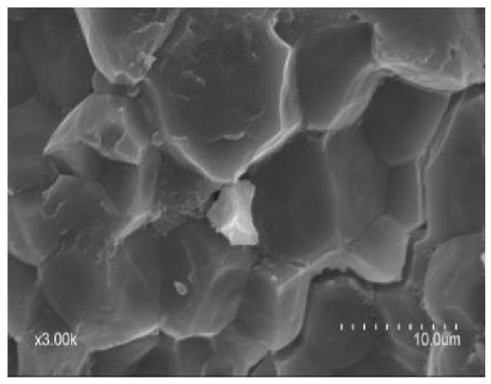

Wild type
B
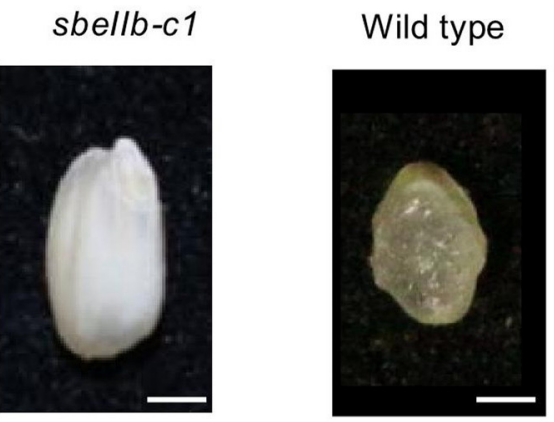

sbel-c6

sbellb-c1
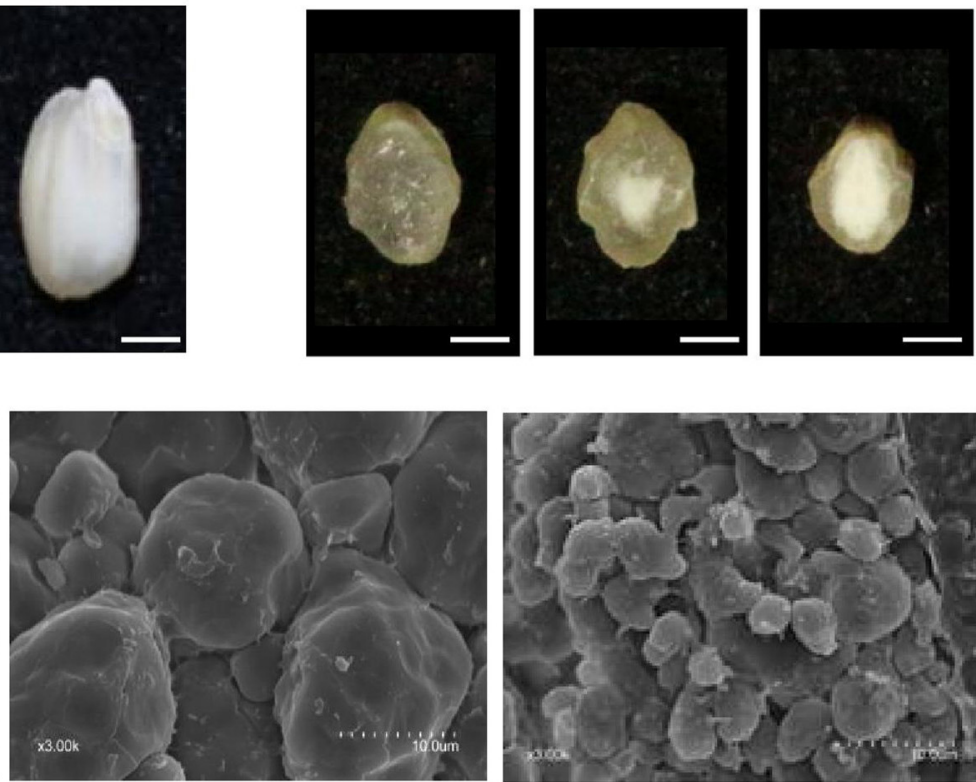

sbel-c6

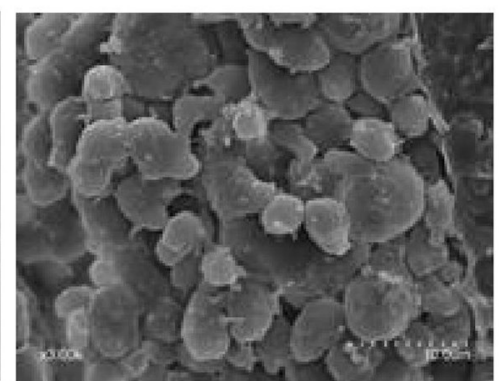

sbellb-c1

FIGURE 3 | Grain and starch granule morphology profiles of the sbel and sbellb mutant lines. (A) Morphologies of the brown grains. Bars = 1 mm.

(B) Transverse sections of grains from wild type, sbel-c6 and sbellb-c1 mutant lines. Bars = $1 \mathrm{~mm}$. (C) Scanning electron microscopy images of the starch in wild type, sbel-c6 and sbellb-c1 mutant grains. Bars $=10 \mu \mathrm{m}$.

TABLE 3 | Grain morphology and starch physiochemical properties of different mutant lines.

\begin{tabular}{|c|c|c|c|c|c|}
\hline Properties & Wild type & sbel-c6 (i1a/i1a) & sbel-c31 (d8/d8) & sbellb-c1 (i1a/i1a) & sbellb-c15 (i1b/i1b) \\
\hline Thousand-grain weight (g) & $24 \pm 0.42 \mathrm{a}$ & $24 \pm 0.45 \mathrm{a}$ & $24 \pm 0.13 a$ & $17 \pm 0.46 b$ & $17 \pm 0.36 b$ \\
\hline Length (mm) & $5.5 \pm 0.10 \mathrm{a}$ & $5.5 \pm 0.10 \mathrm{a}$ & $5.4 \pm 0.10 \mathrm{a}$ & $4.9 \pm 0.10 b$ & $4.8 \pm 0 b$ \\
\hline Width (mm) & $2.5 \pm 0.10 \mathrm{a}$ & $2.5 \pm 0.10 \mathrm{a}$ & $2.4 \pm 0.10 \mathrm{a}$ & $2.0 \pm 0 \mathrm{~b}$ & $2.1 \pm 0 \mathrm{~b}$ \\
\hline Thickness (mm) & $2.0 \pm 0.01 \mathrm{a}$ & $2.0 \pm 0.10 \mathrm{a}$ & $2.0 \pm 0.10 \mathrm{a}$ & $1.8 \pm 0 b$ & $1.7 \pm 0 b$ \\
\hline Chalkiness (\% per grain) & $0-10$ & $0-10$ & $0-10$ & $75-100$ & $75-100$ \\
\hline Hardness & $1.26 \pm 0.01 \mathrm{a}$ & $1.04 \pm 0.02 b$ & $1.10 \pm 0.01 b$ & $0.30 \pm 0.01 c$ & $0.31 \pm 0.01 c$ \\
\hline Gumminess & $1.10 \pm 0.05 a$ & $0.73 \pm 0.06 b$ & $0.76 \pm 0.09 b$ & $0.27 \pm 0.03 c$ & $0.29 \pm 0.02 c$ \\
\hline Chewiness & $8.25 \pm 0.01 \mathrm{a}$ & $6.52 \pm 0.04 b$ & $6.69 \pm 0.06 b$ & $0.15 \pm 0.04 c$ & $0.22 \pm 0.02 c$ \\
\hline Gel consistency & $31 \pm 1$ & $28 \pm 1$ & $28.9 \pm 1$ & $33.2 \pm 0.6$ & $33.1 \pm 0.2$ \\
\hline Onset gelatinization temperature $\left({ }^{\circ} \mathrm{C}\right)$ & $60 \pm 0.40 a$ & $55 \pm 0.61 \mathrm{a}$ & $56.5 \pm 1.10 \mathrm{a}$ & $73.6 \pm 0.92 b$ & $74.5 \pm 1.20 \mathrm{~b}$ \\
\hline Peak gelatinization temperature $\left({ }^{\circ} \mathrm{C}\right)$ & $66.9 \pm 0.80 \mathrm{a}$ & $62.4 \pm 1.20 \mathrm{a}$ & $63.6 \pm 0.70 \mathrm{a}$ & $80.4 \pm 1.40 b$ & $81.2 \pm 2.20 b$ \\
\hline End gelatinization temperature $\left({ }^{\circ} \mathrm{C}\right)$ & $73.9 \pm 1.30 \mathrm{a}$ & $70.1 \pm 1.11 \mathrm{a}$ & $71.2 \pm 0.80 \mathrm{a}$ & $86.7 \pm 1.50 \mathrm{~b}$ & $88.1 \pm 1.80 \mathrm{~b}$ \\
\hline Gelatinization enthalpy (J/g) & $4.5 \pm 0.12 \mathrm{a}$ & $2.68 \pm 0.06 b$ & $2.58 \pm 0.07 b$ & $6.6 \pm 0.10 c$ & $6.7 \pm 0.12 c$ \\
\hline Pentosans (\%) & $1.72 \pm 0.10 \mathrm{a}$ & $1.78 \pm 0.02 \mathrm{a}$ & $1.77 \pm 0.015 a$ & $2.14 \pm 0.08 b$ & $2.22 \pm 0.04 b$ \\
\hline
\end{tabular}

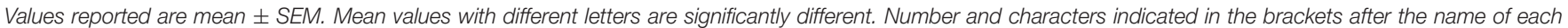
mutant line, i1 and d8, represent insertion of $1 \mathrm{bp}$ or deletion of $8 \mathrm{bp}$ at the target sites, respectively, and i1a or i1 b indicates the 1 bp insertion of different nucleoid base.

lines showed a significantly decrease in hardness, gumminess, and chewiness with more pronounced effects observed in the sbeIIb mutant lines (Table 3). Furthermore, the flours from sbeIIb mutant lines failed to paste at the temperature profile set for RVA analysis, and demonstrated significant decreased values of several viscosity parameters such as the peak viscosity (PV), hot viscosity $(\mathrm{HV})$, breakdown value (BDV, PV-HV), final viscosity
(FV) and setback value (SBV, FV-HV). Whereas those values of the sbeI mutants were similar to those of wild type with only a slight decrease of PV (Figure 6 and Table 3). We further analyzed the thermal properties of starch from both sbeI and sbeIIb mutant lines by differential scanning calorimetry (DSC). The peak temperature indicates the transition induced by melting amylopectin crystallities, whereas the end temperature represents 
A

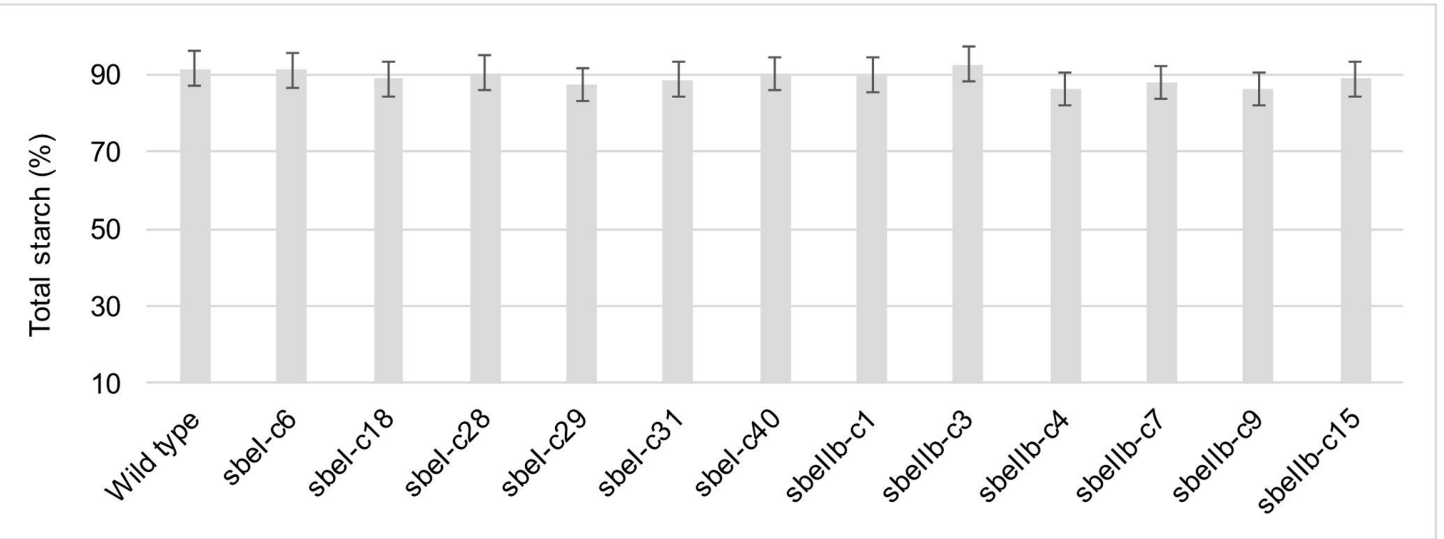

B

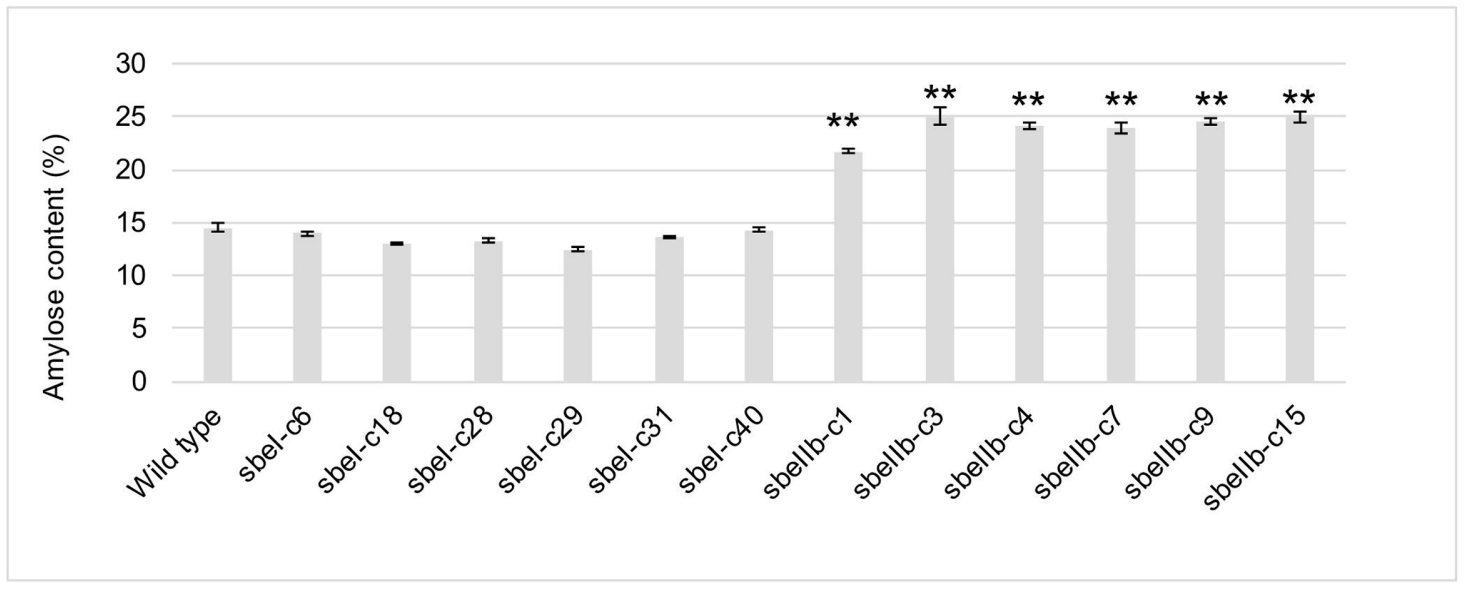

C

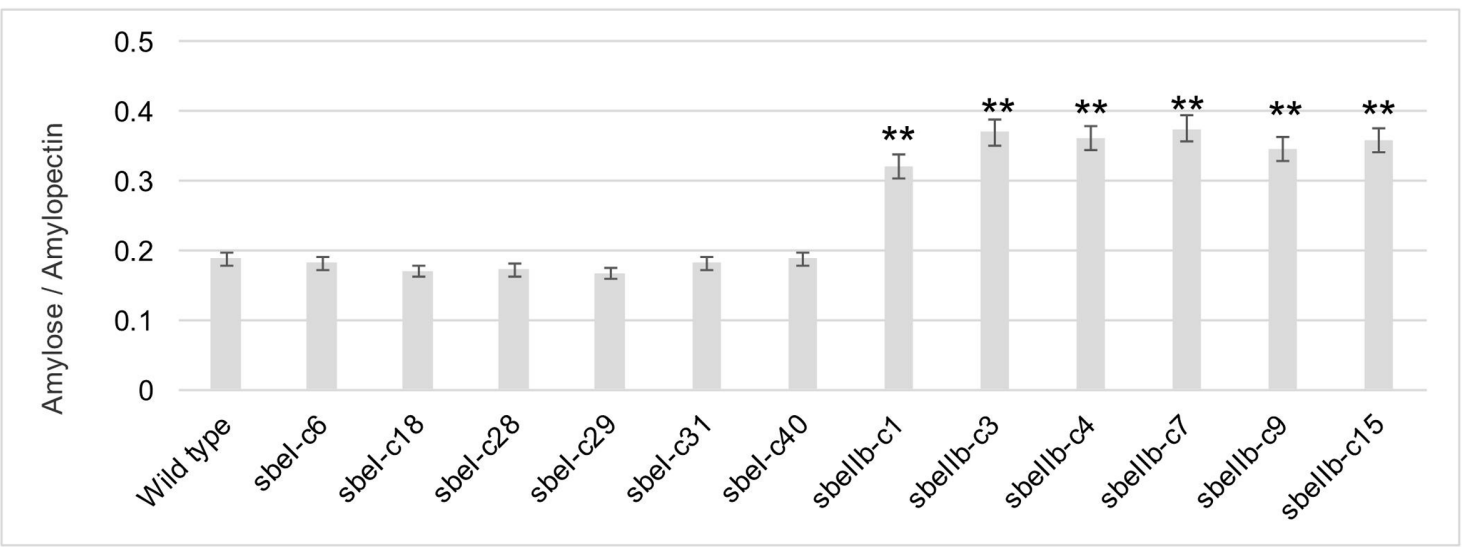

FIGURE 4 | The starch contents of polished grains from different sbel and sbellb mutant lines. (A) Total starch contents of polished grains of different sbel and sbellb mutant lines. (B) Amylose contents (ACs) of polished grains from different mutant lines. (C) Ratios of amylose/amylopectin of the starch in different mutant lines (**Student's $t$-test, $n=3, P<0.01$ ).

the transition of the dissociation of the amylose-lipid complex (Regina et al., 2010). As indicated in Table 3, the onset, peak and end gelatinization temperatures, and gelatinization enthalpy of thermo-gelatinization for the starch of sbeIIb mutant rice lines were significantly increased compared to those of the wild type (Student's $t$-test, ${ }^{* *} P<0.01$ ). In contrast, the sbeI mutant starches had similar gelatinization temperatures and decreased gelatinization enthalpy of thermo-gelatinization compared to those of the wild type (Student's $t$-test, ${ }^{*} P<0.01$ ) (Table 3 ). These results indicated that the flours of the sbeIIb mutant rice lines possessed a stronger resistance to alkaline gelatinization and swelling of its granules. 
A

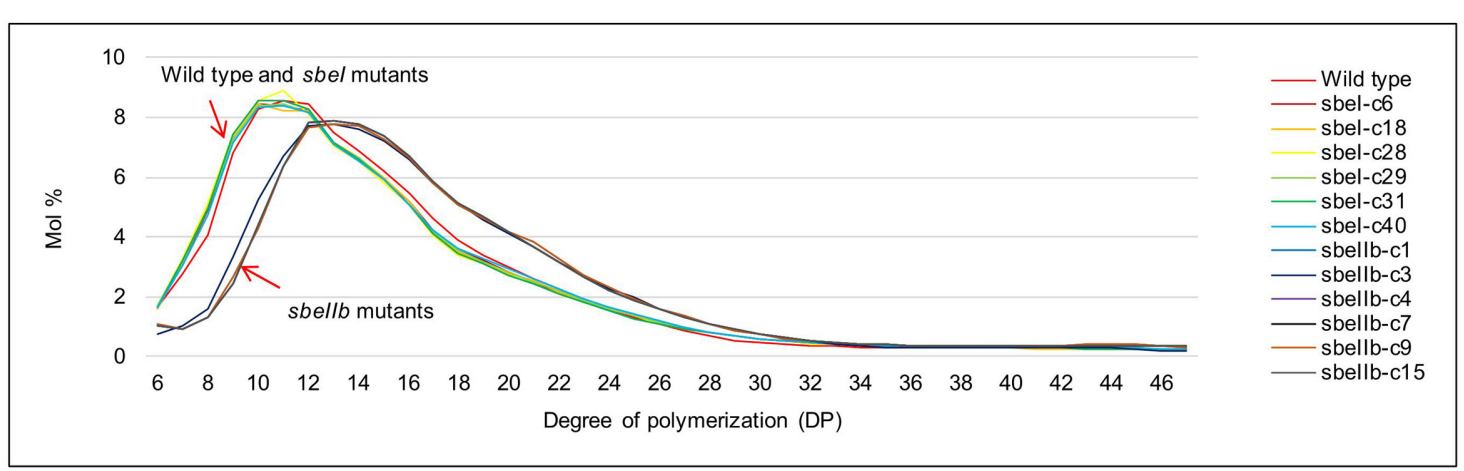

B

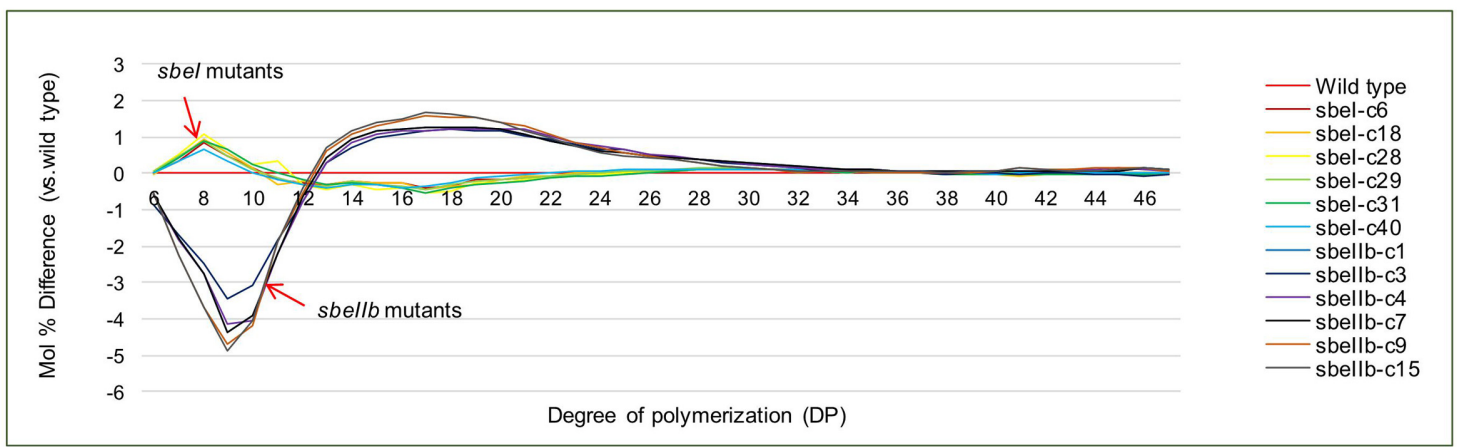

FIGURE 5 | Chain-length distribution profiles of isoamylase debranched starches from different mutant lines. (A) Chain length distribution (CLD) profiles of debranched starch from sbel and sbellb mutant lines compared with wild type. (B) Differences were calculated by subtracting chain-length distributions of isoamylase-debranched wild type from sbel and sbellb mutant lines, respectively.

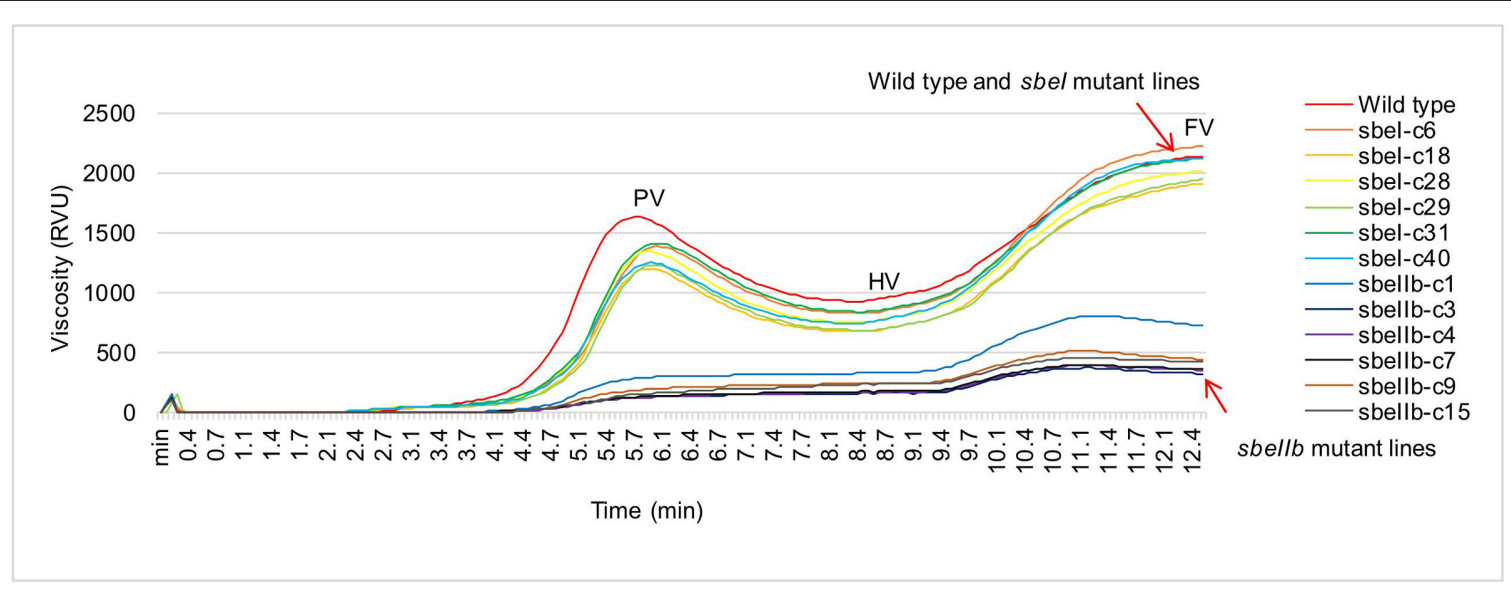

FIGURE 6 | Rapid viscosity profiles of rice flours from different sbe mutant lines. Pasting curves of the flours from sbel and sbelllb mutant lines were measured by a rapid visco analyser (RVA). PV, peak viscosity; HV, hot viscosity; FV, final viscosity.

The in vitro RS content of freshly cooked sbeIIb mutant grains reached as high as $9.8 \%$ when measured following the protocol described by the AOAC Method 2002.02 (McCleary et al., 2002), which is significantly higher than that of wild type and sbeI mutant lines in which little or no RS was detected in the grains (Figure 7A). Furthermore, the content of reducing sugar, which is positively and linearly correlated to GI, was much lower in the grains of sbeIIb mutant lines, while no obvious difference was detected between the sbeI mutant lines and wild type (Figure 7B). At the same time, the content of pentosans, which are perceived as one of the major components of healthy dietary fiber, was also significantly higher in the grains of sbeIIb mutant lines compared to wild type, whereas no obvious difference was observed between sbeI mutant lines and wild type (Table 3 ). 

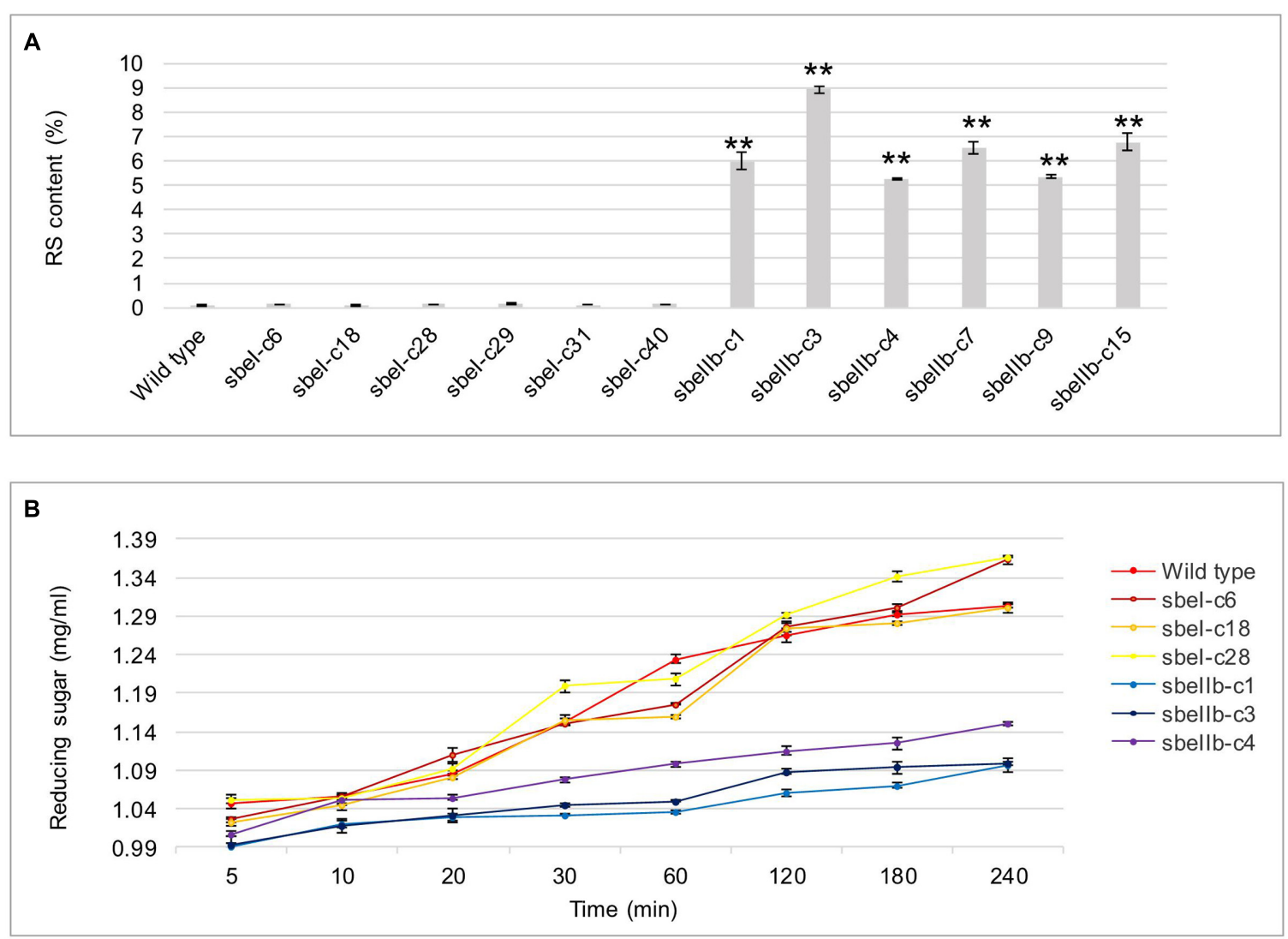

FIGURE 7 | Contents of resistant starch and reducing sugar in the grains from different sbe mutant lines. (A) RS content of grains of mutant lines and wild type. (B) Content of reducing sugar after a-amylase treatment (**Student's $t$-test, $n=3, P<0.01$ ).

\section{DISCUSSION}

In this study, we successfully generated high amylose rice by CRISPR/Cas9-mediated targeted mutagenesis in SBEIIb. We designed gRNAs specifically targeting SBEI and SBEIIb in rice and obtained transgene-free homozygous sbeIIb mutants with a significantly increased AC and RS contents (Figure 7). Compared to conventional breeding, chemical or physical radiation induced mutagenesis and transgene-based strategies, CRISPR/Cas9-mediated genome editing technology can modify a target gene more precisely in a very convenient way (Jones, 2015). No need for laborious crossing and backcrossing and 'clean' plants without transgene could be obtained in one or two generations. Thus, by using a japonica rice variety Kitaake as an example, we here further demonstrated the advantages of CRISPR/Cas9-mediated genome editing in crop improvement and presented a better alternative strategy to breed for high amylose rice to meet increasing demand of people who suffer from diet-related non-infectious chronic diseases.

Reduction of SBEs' activities led to decreased ratio of branch points in the amylopectin fraction and increased AC (Morell et al., 2004). Another effect is on the frequency or distribution of branches (Thompson, 2000; Yao et al., 2004). Whereas SBEIIa and SBEIIb usually generate short chains, SBEI has an apparent function in the formation of long chains of amylopectin (Nishi et al., 2001; Nakamura, 2002; Satoh et al., 2003). Different SBEs play different roles in starch biosynthesis in different plant species. No apparent functionof SBEI was foundin starch metabolism in Arabidopsis (Dumez et al., 2006) and abolishing SBEI activity resulted in no measurable impact on starch structure or functionality in potato, maize, and wheat (Safford et al., 1998; Blauth et al., 2002; Regina et al., 2004). In contrast, a significant increase of AC content was achieved through down-regulation or elimination of SBEII expression in different crop species. For example, down-regulation of SBEII in potato leads to an increase in amylose to approximately 35\% (Jobling et al., 1999), whereas potato starch with an AC of 70\% or higher was yielded by co-suppression of SBEI and SBEII (Schwall et al., 2000). In barley, suppression of both SBEIIa and SBEIIb generated starch with AC higher than 70\% (Regina et al., 2010). In barley, starch granules containing almost entirely amylose were obtained by suppression of all the SBE genes (Carciofi et al., 2012). In maize endosperm, the amylose extender (ae) phenotype with ACs ranged from 50 to $80 \%$ were obtained by lesions in the SBEIIb gene (Garwood et al., 1976), whereas lesions in SBEIIa had no effect on endosperm starch (Blauth et al., 2001). In wheat, suppression of the expression of SBEIIa yielded a $>70 \%$ AC (Regina et al., 2006). In rice, down-regulation of SBEIIb resulted 
in increased AC up to 25-30\% in a japonica background (Mizuno et al., 1993; Nishi et al., 2001). Artificial microRNA and hairpin RNA-mediated RNAi silencing of SBEIIb expression in rice grains resulted in apparently elevated levels of amylose and RS and thus lower GI values (Butardo et al., 2011). Inhibition of SBEI led to unaltered AC. In contrast, the SBEIIb RNAi transgenic rice grains showed a substantial increase in AC. However, the increase of AC was much more significant in both SBEI and SBEIIb antisense RNAs transgenic grains, indicating that simultaneous manipulation of SBEI and SBEIIb genes has the potential to dramatically increase the AC in rice grains (Zhu et al., 2012). Our results are consistent with previous studies. Knock-out of SBEIIb leads to the increase of AC and longer amylopectin chains (Figures 4, 5). In contrast, sbe1 mutant lines and wild type were very similar in terms of AC content (Figure 4) (Mizuno et al., 1993; Nishi et al., 2001; Butardo et al., 2011; Zhu et al., 2012). The sbeIIb mutant lines contained more long chains than wild type, with increased proportion of the long chains (DP $>14$ ) and a concomitant decrease in the ratio of short chains (DP 612) amylopectin (Figure 5). This is in good agreement with an increased proportion of intermediate chains observed in either sbeIIb mutant generated by chemical or physical mutagenesis or sbeIIb RNAi lines (Mizuno et al., 1993; Nishi et al., 2001; Satoh et al., 2003; Butardo et al., 2011; Zhu et al., 2012). As for sbeI mutant lines, a slight increase in the ratio of amylopectin short chains (DP 6-12) accompanied by a decrease in the long chains $(\mathrm{DP}>14$ ) was observed (Figure 5), consistent with findings from the previous sbe1 rice mutant, which was generated through chemical mutagen, and had an altered fine structure of amylopectin caused by a significant decrease in chains of DP 12 to 21 , and an increase in DP $<12$ (Satoh et al., 2003). However, our results further indicated that rice SBEI plays a different role compared to $S B E I$ genes in potato, maize, and wheat, in which the inactivation of SBEI resulted in no major impact on amylopectin structure and starch properties (Safford et al., 1998; Blauth et al., 2002; Regina et al., 2004).

It has been demonstrated that the higher proportion of long chains of amylopectin in starch granules led to resistance to gelatinization (Bhattacharyya et al., 1990; Mizuno et al., 1993; Stinard et al., 1993; Safford et al., 1998; Wang et al., 1998; Jane et al., 1999; Nishi et al., 2001). Our results showed that the onset, peak, and end temperature of thermo-gelatinization of the endosperm starches of sbeIIb mutants were significantly increased compared to those of wild type (Table 3 ). In contrast, the endosperm starches from the sbeI mutants had similar onset, peak, and end temperatures with those of wild type (Table 3), indicating that not only the increased of AC led to the suppression of hydration and swelling of starch, but also the modification of amylopectin fine structure affects physicochemical properties of starch. Furthermore, compared with wild type, an apparent increased RS was observed in the sbeIIb mutant lines (Figure 7). Moreover, the sbeIIb mutant lines also had an increased content of pentosan and decreased level of reducing sugar in comparison to wild type (Table 3 and Figure 7), as observed in SBEIIb RNAi lines in rice (Butardo et al., 2011). Interestingly, slight differences existed in the RVA profiles between $s b e I I b-c 1$ and those of the rest sbeIIb lines (Figure 6), and RS contents between sbeIIb-c3 and the rest of sbeIIb mutant lines (Figure 7), as observed in the fragrant rice generated by TALEN technology (Shan et al., 2015). The reason for this remains unclear; however, it implies that it is important to screen the mutant lines with the highest RS content and lowest reducing sugar content in a breeding program. Finally, it is worth to note that although the altered the fine structure of amylopectin and slight decreased resistance to thermo-gelatinization were observed in our sbeI mutant lines and in a previous sbeI mutant (Satoh et al., 2003), the proportion of RS and level of reducing sugar remained at levels similar to those of wild type (Table 3 and Figure 7). These results suggest that except for amylose, longer and intermediate chain amylopectin molecules may also have an effect on property of the starch molecules, especially the elevations in the ratio of intermediate chains (DP > 14) are associated with increased level of RS in rice (Butardo et al., 2011).

Taken together, we here not only demonstrated the feasibility to create transgene-free high amylose rice plants through CRISPR/Cas9-mediated editing of SBEIIb, but also further defined that SBEIIb plays a more important role than SBEI in determining the fine structure of amylopectin and nutritional properties of starch in rice grain, and thus could be employed as a primary target gene for generating high-amylose rice to meet the increasing demand from people affected by diet-related non-infectious chronic diseases.

\section{AUTHOR CONTRIBUTIONS}

LX and YZ conceived and designed the experiments; YS, GJ, ZL, $\mathrm{XZ}$, JL, XG, WD, and JD performed the experiments; YS and GJ analyzed the data; LX and YS wrote the manuscript; YZ and FF revised the manuscript. All authors read and approved the final manuscript.

\section{ACKNOWLEDGMENTS}

This work is partly funded by the Ministry of Science and Technology of China (grant no. 2016YFD0100500) and the Ministry of Agriculture of China (grant no. 2016ZX08010-003). YS is supported by a GSCAAS-ULg Joint Ph.D. Program.

\section{SUPPLEMENTARY MATERIAL}

The Supplementary Material for this article can be found online at: http://journal.frontiersin.org/article/10.3389/fpls.2017.00298/ full\#supplementary-material 


\section{REFERENCES}

AACC (1995). Approved Methods of the American Association of Cereal Chemists, 9th Edn. St. Paul, MN: AACC.

Asaoka, M., Okuno, K., Sugimoto, Y., Yano, M., Omura, T., and Fuwa, H. (1986). Characterization of endosperm starch from high-amylose mutants of rice (Oryza sativa L.). Starch 38, 114-117. doi: 10.1002/star.198603 80403

Asp, N.-G. (1992). Resistant starch: proceedings from the 2nd plenary meeting of EURESTA: European Flair Concerted Action no. 11 (COST 911): physiological implications of the consumption of resistant starch in man. Eur. J. Clin. Nutr. 46, S1-S148. doi: 10.1016/0924-2244(92)90153-n

Ball, S. G., and Morell, M. K. (2003). From bacterial glycogen to starch: understanding the biogenesis of the plant starch granule. Annu. Rev. Plant Biol. 54, 207-233. doi: 10.1146/annurev.arplant.54.031902. 134927

Bhattacharyya, M. K., Smith, A. M., Ellis, T. N., Hedley, C., and Martin, C. (1990). The wrinkled-seed character of pea described by Mendel is caused by a transposon-like insertion in a gene encoding starch-branching enzyme. Cell 60, 115-122. doi: 10.1016/0092-8674(90)90721-P

Blauth, S. L., Kim, K.-N., Klucinec, J., Shannon, J. C., Thompson, D., and Guiltinan, M. (2002). Identification of mutator insertional mutants of starchbranching enzyme 1 (sbe1) in Zea mays L. Plant Mol. Biol. 48, 287-297. doi: 10.1023/A:1013335217744

Blauth, S. L., Yao, Y., Klucinec, J. D., Shannon, J. C., Thompson, D. B., and Guiltinan, M. J. (2001). Identification of Mutator insertional mutants of starchbranching enzyme 2a in corn. Plant Physiol. 125, 1396-1405. doi: 10.1104/pp. 125.3.1396

Boyer, C. D., and Preiss, J. (1978). Multiple forms of $(1 \rightarrow 4)-\alpha-D$ glucan, $(1 \rightarrow 4)$ - $\alpha$-D-glucan-6-glycosyl transferase from developing Zea mays L. kernels. Carbohydr. Res. 61, 321-334. doi: 10.1016/S0008-6215(00) 84492-4

Butardo, V. Jr., Fitzgerald, M. A., Rahman, S., and Gidley, M. J. (2008). "Efforts to capture high amylose in rice," in Proceedings of the AACC International Annual Meeting 2008 (Eagan, MN: American Association of Cereal Chemists), A15-A15.

Butardo, V. M., Fitzgerald, M. A., Bird, A. R., Gidley, M. J., Flanagan, B. M., Larroque, O., et al. (2011). Impact of down-regulation of starch branching enzyme IIb in rice by artificial microRNA-and hairpin RNA-mediated RNA silencing. J. Exp. Bot. 62, 4927-4941. doi: 10.1093/jxb/err188

Carciofi, M., Blennow, A., Jensen, S. L., Shaik, S. S., Henriksen, A., Buléon, A., et al. (2012). Concerted suppression of all starch branching enzyme genes in barley produces amylose-only starch granules. BMC Plant Biol. 12:223. doi: 10.1186/1471-2229-12-223

Chen, L., Magliano, D. J., and Zimmet, P. Z. (2012). The worldwide epidemiology of type 2 diabetes mellitus-present and future perspectives. Nat. Rev. Endocrinol. 8, 228-236. doi: 10.1038/nrendo.2011.183

Cong, L., Ran, F. A., Cox, D., Lin, S., Barretto, R., Habib, N., et al. (2013). Multiplex genome engineering using CRISPR/Cas systems. Science 339, 819-823. doi: $10.1126 /$ science. 1231143

Dumez, S., Wattebled, F., Dauvillee, D., Delvalle, D., Planchot, V., Ball, S. G., et al. (2006). Mutants of Arabidopsis lacking starch branching enzyme II substitute plastidial starch synthesis by cytoplasmic maltose accumulation. Plant Cell 18, 2694-2709. doi: 10.1105/tpc.105.037671

Endo, M., Mikami, M., and Toki, S. (2016). Biallelic gene targeting in rice. Plant Physiol. 170, 667-677. doi: 10.1104/pp.15.01663

Englyst, H. N., Kingman, S., and Cummings, J. (1992). Classification and measurement of nutritionally important starch fractions. Eur. J. Clin. Nutr. 46, S33-S50.

Feng, Z., Mao, Y., Xu, N., Zhang, B., Wei, P., Yang, D. L., et al. (2014). Multigeneration analysis reveals the inheritance, specificity, and patterns of CRISPR/Cas-induced gene modifications in Arabidopsis. Proc. Natl. Acad. Sci. U.S.A. 111, 4632-4637. doi: 10.1073/pnas.1400822111

Food and Agriculture Organization [FAO] (2004). International Year of Rice (Online). Available at: http://www.fao.org/rice2004/en/rice-us.htm (accessed March 13, 2006)

Gao, X., Chen, J., Dai, X., Zhang, D., and Zhao, Y. (2016). An effective strategy for reliably isolating heritable and Cas9-free Arabidopsis mutants generated by
CRISPR/Cas9-mediated genome editing. Plant Physiol. 171, 1794-1800. doi: 10.1104/pp.16.00663

Gao, Y., and Zhao, Y. (2014). Self-processing of ribozyme-flanked RNAs into guide RNAs in vitro and in vivo for CRISPR-mediated genome editing. J. Integr. Plant Biol. 56, 343-349. doi: 10.1111/jipb.12152

Garwood, D. L., Shannon, J., and Creech, R. (1976). Starches of endosperms possessing different alleles at the amylose-extender locus in Zea mays L. [maize]. Cereal Chem. 53, 355-364.

Giacco, R., Clemente, G., Brighenti, F., Mancini, M., D’avanzo, A., Coppola, S., et al. (1998). Metabolic effects of resistant starch in patients with type 2 diabetes. Diabetes Nutr. Metab. 11, 330-335.

Hanashiro, I., Abe, J.-I., and Hizukuri, S. (1996). A periodic distribution of the chain length of amylopectin as revealed by high-performance anion-exchange chromatography. Carbohydr. Res. 283, 151-159. doi: 10.1016/0008-6215(95) 00408-4

Hiei, Y., Ohta, S., Komari, T., and Kumashiro, T. (1994). Efficient transformation of rice (Oryza sativa L.) mediated by Agrobacterium and sequence analysis of the boundaries of the T-DNA. Plant J. 6, 271-282. doi: 10.1046/j.1365-313X. 1994.6020271.x

Hoebler, C., Karinthi, A., Chiron, H., Champ, M., and Barry, J. (1999). Bioavailability of starch in bread rich in amylose: metabolic responses in healthy subjects and starch structure. Eur. J. Clin. Nutr. 53, 360-366. doi: 10.1038/sj. ejcn. 1600718

Itoh, K., Ozaki, H., Okada, K., Hori, H., Takeda, Y., and Mitsui, T. (2003). Introduction of $\mathrm{Wx}$ transgene into rice wx mutants leads to both highand low-amylose rice. Plant Cell Physiol. 44, 473-480. doi: 10.1093/pcp/ pcg068

Jane, J., Chen, Y., Lee, L., Mcpherson, A., Wong, K., Radosavljevic, M., et al. (1999). Effects of amylopectin branch chain length and amylose content on the gelatinization and pasting properties of starch 1. Cereal Chem. 76, 629-637. doi: 10.1094/CCHEM.1999.76.5.629

Jiang, H., Lio, J., Blanco, M., Campbell, M., and Jane, J. L. (2010) Resistant-starch formation in high-amylose maize starch during kernel development. J. Agric. Food Chem. 58, 8043-8047. doi: 10.1021/jf1 $01056 y$

Jinek, M., Chylinski, K., Fonfara, I., Hauer, M., Doudna, J. A., and Charpentier, E. (2012). A programmable dual-RNA-guided DNA endonuclease in adaptive bacterial immunity. Science 337, 816-821. doi: 10.1126/science. 1225829

Jobling, S. A., Schwall, G. P., Westcott, R. J., Sidebottom, C. M., Debet, M., Gidley, M. J., et al. (1999). A minor form of starch branching enzyme in potato (Solanum tuberosum L.) tubers has a major effect on starch structure: cloning and characterisation of multiple forms of SBE A. Plant J. 18, 163-171. doi: 10.1046/j.1365-313X.1999.00441.X

Jones, H. D. (2015). Regulatory uncertainty over genome editing. Nat. Plants 1, 14011. doi: 10.1038/nplants.2014.11

Kim, K.-S., Hwang, H.-G., Kang, H.-J., Hwang, I.-K., Lee, Y.-T., and Choi, H.-C. (2005). Ultrastructure of individual and compound starch granules in isolation preparation from a high-quality, low-amylose rice, ilpumbyeo, and its mutant, G2, a high-dietary fiber, high-amylose rice. J. Agric. Food Chem. 53, 8745-8751. doi: 10.1021/jf051194a

Li, C., and Gilbert, R. G. (2016). Progress in controlling starch structure by modifying starch-branching enzymes. Planta 243, 13-22. doi: 10.1007/s00425015-2421-2

Li, J., Sun, Y., Du, J., Zhao, Y., and Xia, L. (2016). Generation of targeted point mutations in rice by a modified CRISPR/Cas9 system. Mol. Plant doi: 10.1016/ j.molp.2016.12.001 [Epub ahead of print].

Li, M., Li, X., Zhou, Z., Wu, P., Fang, M., Pan, X., et al. (2016). Reassessment of the four yield-related Genes Gn1a, DEP1, GS3, and IPA1 in rice using a CRISPR/Cas9 system. Front. Plant Sci. 7:377. doi: 10.3389/fpls.2016. 00377

Li, Z., Liu, Z.-B., Xing, A., Moon, B. P., Koellhoffer, J. P., Huang, L., et al. (2015). Cas9-guide RNA directed genome editing in soybean. Plant Physiol. 169, 960-970. doi: 10.1104/pp.15.00783

Ma, X., Zhang, Q., Zhu, Q., Liu, W., Chen, Y., Qiu, R., et al. (2015). A robust CRISPR/Cas9 system for convenient, high-efficiency multiplex genome editing in monocot and dicot plants. Mol. Plant 8, 1274-1284. doi: 10.1016/j.molp.2015. 04.007 
McCleary, B. V., McNally, M., and Rossiter, P. (2002). Measurement of resistant starch by enzymatic digestion in starch and selected plant materials: collaborative study. J. AOAC Int. 85, 1103-1111.

Mizuno, K., Kawasaki, T., Shimada, H., Satoh, H., Kobayashi, E., Okumura, S., et al. (1993). Alteration of the structural properties of starch components by the lack of an isoform of starch branching enzyme in rice seeds. J. Biol. Chem. 268, 19084-19091.

Mizuno, K., Kimura, K., Arai, Y., Kawasaki, T., Shimada, H., and Baba, T. (1992). Starch branching enzymes from immature rice seeds. J. Biochem. 112, 643-651. doi: 10.1093/oxfordjournals.jbchem.a123953

Morell, M. K., Konik-Rose, C., Ahmed, R., Li, Z., and Rahman, S. (2004). Synthesis of resistant starches in plants. J. AOAC Int. 87, 740-748.

Morell, M. K., and Myers, A. M. (2005). Towards the rational design of cereal starches. Curr. Opin. Plant Biol. 8, 204-210. doi: 10.1016/j.pbi.2005.01.009

Nakamura, Y. (2002). Towards a better understanding of the metabolic system for amylopectin biosynthesis in plants: rice endosperm as a model tissue. Plant Cell Physiol. 43, 718-725. doi: 10.1093/pcp/pcf091

Nakamura, Y., Takeichi, T., Kawaguchi, K., and Yamanouchi, H. (1992). Purification of two forms of starch branching enzyme (Q-enzyme) from developing rice endosperm. Physiol. Plant 84, 329-335. doi: 10.1111/j.13993054.1992.tb04672.x

Nishi, A., Nakamura, Y., Tanaka, N., and Satoh, H. (2001). Biochemical and genetic analysis of the effects of amylose-extender mutation in rice endosperm. Plant Physiol. 127, 459-472. doi: 10.1104/pp.010127

Ohdan, T., Francisco, P. B., Sawada, T., Hirose, T., Terao, T., Satoh, H., et al. (2005). Expression profiling of genes involved in starch synthesis in sink and source organs of rice. J. Exp. Bot. 56, 3229-3244. doi: 10.1093/jxb/ eri292

Ossowski, S., Schwab, R., and Weigel, D. (2008). Gene silencing in plants using artificial microRNAs and other small RNAs. Plant J. 53, 674-690. doi: 10.1111/ j.1365-313X.2007.03328.x

Paul, J. W. III., and Qi, Y. (2016). CRISPR/Cas9 for plant genome editing: accomplishments, problems and prospects. Plant Cell Rep. 35, 1417-1427. doi: 10.1007/s00299-016-1985-z

Puchta, H., and Fauser, F. (2014). Synthetic nucleases for genome engineering in plants: prospects for a bright future. Plant J. 78, 727-741. doi: 10.1111/tpj. 12338

Rahman, S., Bird, A., Regina, A., Li, Z., Ral, J. P., Mcmaugh, S., et al. (2007). Resistant starch in cereals: exploiting genetic engineering and genetic variation. J. Cereal Sci. 46, 251-260. doi: 10.1016/j.jcs.2007.05.001

Regina, A., Bird, A., Topping, D., Bowden, S., Freeman, J., Barsby, T., et al. (2006). High-amylose wheat generated by RNA interference improves indices of large-bowel health in rats. Proc. Natl. Acad. Sci. U.S.A. 103, 3546-3551. doi: 10.1073/pnas.0510737103

Regina, A., Kosar-Hashemi, B., Li, Z., Rampling, L., Cmiel, M., Gianibelli, M. C., et al. (2004). Multiple isoforms of starch branching enzyme-I in wheat: lack of the major SBE-I isoform does not alter starch phenotype. Funct. Plant Biol. 31, 591-601. doi: 10.1071/FP03193

Regina, A., Kosar-Hashemi, B., Ling, S., Li, Z., Rahman, S., and Morell, M. (2010). Control of starch branching in barley defined through differential RNAi suppression of starch branching enzyme IIa and IIb. J. Exp. Bot. 61, 1469-1482. doi: $10.1093 /$ jxb/erq011

Regmi, P. R., Metzler-Zebeli, B. U., Gänzle, M. G., Van Kempen, T. A., and Zijlstra, R. T. (2011). Starch with high amylose content and low in vitro digestibility increases intestinal nutrient flow and microbial fermentation and selectively promotes bifidobacteria in pigs. J. Nutr. 141, 1273-1280. doi: 10.3945/jn.111. 140509

Safford, R., Jobling, S. A., Sidebottom, C. M., Westcott, R. J., Cooke, D., Tober, K. J., et al. (1998). Consequences of antisense RNA inhibition of starch branching enzyme activity on properties of potato starch. Carbohydr. Polym. 35, 155-168. doi: 10.1016/S0144-8617(97)00249-X

Satoh, H., Nishi, A., Yamashita, K., Takemoto, Y., Tanaka, Y., Hosaka, Y., et al. (2003). Starch-branching enzyme I-deficient mutation specifically affects the structure and properties of starch in rice endosperm. Plant Physiol. 133, 1111-1121. doi: 10.1104/pp.103.021527

Schwall, G. P., Safford, R., Westcott, R. J., Jeffcoat, R., Tayal, A., Shi, Y.-C., et al. (2000). Production of very-high-amylose potato starch by inhibition of SBE A and B. Nat. Biotechnol. 18, 551-554. doi: 10.1038/75427
Shan, Q., Wang, Y., Li, J., Zhang, Y., Chen, K., Liang, Z., et al. (2013). Targeted genome modification of crop plants using a CRISPR-Cas system. Nat. Biotechnol. 31, 686-688. doi: 10.1038/nbt.2650

Shan, Q., Zhang, Y., Chen, K., Zhang, K., and Gao, C. (2015). Creation of fragrant rice by targeted knockout of the OsBADH2 gene using TALEN technology. Plant Biotechnol. J. 13, 791-800. doi: 10.1111/pbi.12312

Shi, J., Gao, H., Wang, H., Lafitte, H. R., Archibald, R. L., Yang, M., et al. (2016). ARGOS8 variants generated by CRISPR-Cas9 improve maize grain yield under field drought stress conditions. Plant Biotechnol. J. 15, 207-216. doi: 10.1111/ pbi. 12603

Shu, X., Jiao, G., Fitzgerald, M. A., Yang, C., Shu, Q., and Wu, D. (2006). Starch structure and digestibility of rice high in resistant starch. Starch 58, 411-417. doi: 10.1002/star.200600501

Stinard, P. S., Robertson, D. S., and Schnable, P. S. (1993). Genetic isolation, cloning, and analysis of a mutator-induced, dominant antimorph of the maize amylose extender1 locus. Plant Cell 5, 1555-1566. doi: 10.1105/tpc.5.11.1555

Sun, Y., Li, J., and Xia, L. (2016a). Precise genome modification via sequencespecific nucleases-mediated gene targeting for crop improvement. Front. Plant Sci. 7:1928. doi: 10.3389/fpls.2016.01928

Sun, Y., Zhang, X., Wu, C., He, Y., Ma, Y., Hou, H., et al. (2016b). Engineering herbicide-resistant rice plants through CRISPR/Cas9-mediated homologous recombination of acetolactate synthase. Mol. Plant 9, 628-631. doi: 10.1016/j. molp.2016.01.001

Svitashev, S., Young, J. K., Schwartz, C., Gao, H., Falco, S. C., and Cigan, A. M. (2015). Targeted mutagenesis, precise gene editing, and site-specific gene insertion in maize using Cas9 and guide RNA. Plant Physiol. 169, 931-945. doi: $10.1104 /$ pp. 15.00793

Syahariza, Z., Sar, S., Hasjim, J., Tizzotti, M. J., and Gilbert, R. G. (2013). The importance of amylose and amylopectin fine structures for starch digestibility in cooked rice grains. Food Chem. 136, 742-749. doi: 10.1016/j.foodchem.2012. 08.053

Thompson, D. (2000). On the non-random nature of amylopectin branching. Carbohydr. Polym. 43, 223-239. doi: 10.1016/S0144-8617(00)00150-8

Unwin, N., Whiting, D., and Guariguata, L. (2013). International Diabetes Federation. IDF Diabetes Atlas. Brussels: International Diabetes Federation, 2012.

Vonk, R. J., Hagedoorn, R. E., De Graaff, R., Elzinga, H., Tabak, S., Yang, Y.-X., et al. (2000). Digestion of so-called resistant starch sources in the human small intestine. Am. J. Clin. Nutr. 72, 432-438.

Voytas, D. F., and Gao, C. (2014). Precision genome engineering and agriculture: opportunities and regulatory challenges. PLoS Biol. 12:e1001877. doi: 10.1371/ journal.pbio.1001877

Wang, T. L., Bogracheva, T. Y., and Hedley, C. L. (1998). Starch: as simple as A. B, C? J. Exp. Bot. 49, 481-502. doi: 10.1093/jxb/49.320.481

Wang, Z.-P., Xing, H.-L., Dong, L., Zhang, H.-Y., Han, C.-Y., Wang, X.-C., et al. (2015). Egg cell-specific promoter-controlled CRISPR/Cas9 efficiently generates homozygous mutants for multiple target genes in Arabidopsis in a single generation. Genome Biol. 16, 144. doi: 10.1186/s13059-015-0715-0

Warthmann, N., Chen, H., Ossowski, S., Weigel, D., and Hervé, P. (2008). Highly specific gene silencing by artificial miRNAs in rice. PLOS ONE 3:e1829. doi: 10.1371/journal.pone.0001829

Wei, C., Qin, F., Zhou, W., Chen, Y., Xu, B., Wang, Y., et al. (2010). Formation of semi-compound C-type starch granule in high-amylose rice developed by antisense RNA inhibition of starch-branching enzyme. J. Agric. Food Chem. 58, 11097-11104. doi: 10.1021/jf1024533

Xie, K., Minkenberg, B., and Yang, Y. (2015). Boosting CRISPR/Cas9 multiplex editing capability with the endogenous tRNA-processing system. Proc. Natl. Acad. Sci. U.S.A. 112, 3570-3575. doi: 10.1073/pnas.1420294112

Yamakawa, H., Hirose, T., Kuroda, M., and Yamaguchi, T. (2007). Comprehensive expression profiling of rice grain filling-related genes under high temperature using DNA microarray. Plant Physiol. 144, 258-277. doi: 10.1104/pp.107. 098665

Yamanouchi, H., and Nakamura, Y. (1992). Organ specificity of isoforms of starch branching enzyme (Q-enzyme) in rice. Plant Cell Physiol. 33, 985-991. doi: 10.1111/j.1744-7909.2008.00714.x

Yano, M., Okuno, K., Kawakami, J., Satoh, H., and Omura, T. (1985). High amylose mutants of rice, Oryza sativa L. Theor. Appl. Genet. 69, 253-257. doi: 10.1007/ BF00662436 
Yao, Y., Thompson, D. B., and Guiltinan, M. J. (2004). Maize starch-branching enzyme isoforms and amylopectin structure. In the absence of starch-branching enzyme IIb, the further absence of starch-branching enzyme Ia leads to increased branching. Plant Physiol. 136, 3515-3523. doi: 10.1104/pp.104.043315

Zhou, H., Liu, B., Weeks, D. P., Spalding, M. H., and Yang, B. (2014). Large chromosomal deletions and heritable small genetic changes induced by CRISPR/Cas9 in rice. Nucleic Acids Res. 42, 10903-10914. doi: 10.1093/nar/ gku806

Zhu, L., Gu, M., Meng, X., Cheung, S. C., Yu, H., Huang, J., et al. (2012). Highamylose rice improves indices of animal health in normal and diabetic rats. Plant Biotechnol. J. 10, 353-362. doi: 10.1111/j.1467-7652.2011.00667.x
Conflict of Interest Statement: The authors declare that the research was conducted in the absence of any commercial or financial relationships that could be construed as a potential conflict of interest.

Copyright (C) 2017 Sun, Jiao, Liu, Zhang, Li, Guo, Du, Du, Francis, Zhao and Xia. This is an open-access article distributed under the terms of the Creative Commons Attribution License (CC BY). The use, distribution or reproduction in other forums is permitted, provided the original author(s) or licensor are credited and that the original publication in this journal is cited, in accordance with accepted academic practice. No use, distribution or reproduction is permitted which does not comply with these terms. 